\title{
A co-infection model for Oncogenic HPV and TB with Optimal Control and Cost-Effectiveness Analysis
}

\author{
A. Omame ${ }^{a}$, D. Okuonghae $e^{b, \dagger}$
}

\begin{abstract}
A co-infection model for oncogenic Human papillomavirus (HPV) and Tuberculosis (TB), with optimal control and cost-effectiveness analysis is studied and analyzed to assess the impact of controls against incident infection and against infection with HPV by TB infected individuals as well as optimal TB treatment in reducing the burden of the co-infection of the two diseases in a population. The co-infection model is shown to exhibit the dynamical property of backward bifurcation when the associated reproduction number is less than unity. Furthermore, it is shown that TB and HPV re-infection parameters $\left(\varphi_{\mathrm{P}} \neq 0\right.$ and $\left.\sigma_{\mathrm{T}} \neq 0\right)$ as well as TB exogenous re-infection term $\left(\varepsilon_{1} \neq 0\right)$ induced the phenomenon of backward bifurcation in the oncogenic HPV-TB co-infection model. The global asymptotic stability of the disease-free equilibrium of the co-infection model is also proven not to exist, when the associated reproduction number is below unity. The necessary conditions for the existence of optimal control and the optimality system for the co-infection model is established using the Pontryagin's Maximum Principle. Uncertainty and global sensitivity analysis are also carried out to determine the top ranked parameters that drive the dynamics of the co-infection model, when the associated reproduction numbers as well as the infected populations are used as response functions. Numerical simulations of the optimal control model reveal that the intervention strategy which combines and implements control against HPV infection by TB infected individuals as well as TB treatment control for dually infected individuals is the most cost-effective of all the control strategies for the control and management of the burden of oncogenic HPV and TB co-infection.
\end{abstract}

Keywords: Human Papillomavirus, tuberculosis, co-infection, optimal control, cost-effectiveness analysis.

$\dagger$ Corresponding author: daniel.okuonghae@uniben.edu;danny.okuonghae@corpus-christi.oxon.org 
medRxiv preprint doi: https://doi.org/10.1101/2020.09.15.20195297; this version posted September 27, 2020. The copyright holder for this preprint (which was not certified by peer review) is the author/funder, who has granted medRxiv a license to display the preprint in

It is made available under a CC-BY-NC-ND 4.0 International license .

\section{Introduction}

Tuberculosis (TB), caused by Mycobacterium tuberculosis (MTB), remains a global dominant cause of mortality and morbidity, although treatment and preventive measures are available [24, 38]. Globally, TB is one of the dominant cause of mortality from a single infectious disease agent, responsible for roughly $40 \%$ of deaths [36]. Worldwide, approximately, 10 million new cases of TB and about 1.57 million TB-induced deaths were reported in 2017, with incidence and mortality highest in South-Eastern Asia and Africa [36]. Estimates have equally shown that about one-fourth of the world's population is infected with latent TB and is at the risk of progression to active TB [15].

Cancer is the major cause of mortality in developed countries in Europe and America and the second leading cause of death in developing countries [12. Epidemiological evidences have proven that $80 \%$ of global cancer cases are attributed to the oncogenic Human papillomavirus (HPV) [2]. In a study carried out by Zetola et al. [37, it was revealed that prior TB infection is mostly common among patients with cases of cervical cancer. Likewise, Zhao et al [39], in another study, showed that TB infection is associated with high vulnerability to oncogenic HPV infection. According to Zetola et al. [37], oncogenic HPV and tuberculosis always co-exist, and the immune suppression caused by cancer (which is a consequence of oncogenic HPV) can result in latent TB re-activation, hence, leading to high mortality. In addition, persistent gynecologic TB infection, resulting in chronic inflammation, could be a high risk factor in the progression of oncogenic HPV infection to cervical cancer [37, 39].

Mathematical models have recently been studied to understand the transmission dynamics of human papillomavirus infection (See Omame et al. [23] and the references included therein). Malik et al. [17] investigated an optimal control model for HPV, incorporating optimal vaccination strategies for females in the population. In particular, they considered when three vaccines are used at the same time in comparison to the case where the bivalent cervarix and quadrivalent Gardasil 4 vaccines were used at the initial stage and then, during the course of the vaccination program, one or two of them are interchanged with the nonavalent Gardasil 9 vaccine. Saldana et al. [29] developed and analyzed a two-sex model of HPV with optimal control analysis, incorporating vaccination of adolescents, adults, and screening. They showed that to optimally curb the spread of HPV in a population, vaccination should be administered both before and after the commencement of active sexual life for both females and males in the population. Likewise, TB only models have been extensively studied in the literature (see for instance, [10, 21, 22, 33] and many others).

Lately, mathematical models have been developed to consider the optimal control strategies for the dynamics of infectious diseases including their co-infections [1, 9, 11, 20, 26, 27, 31, 32, 34]. Agusto and Adekunle [1] studied the optimal control and cost-effectiveness analysis of the co-infection of drugresistant tuberculosis and HIV/AIDS. They considered different control strategies for the control of the co-infections of both diseases. They showed that the strategy which combines efforts on minimizing the number of individuals with drug-sensitive and drug-resistant TB and case finding, prevention 
medRxiv preprint doi: https://doi.org/10.1101/2020.09.15.20195297; this version posted September 27, 2020. The copyright holder for this preprint (which was not certified by peer review) is the author/funder, who has granted medRxiv a license to display the preprint in

It is made available under a CC-BY-NC-ND 4.0 International license .

of treatment failure in the drug-sensitive TB infectious individuals and the treatment of individuals with drug-resistant TB is the most cost-effective in curbing the co-infections of drug-resistant TB and HIV/AIDS. Okosun and Makinde [20] considered a malaria-cholera co-infection model, in the presence of prevention and treatment controls for both diseases. Applying the Pontryagin's Maximum principle, they derived the necessary conditions for optimality and equally showed using simulations, that to successfully curb malaria spread, control strategies must include cholera intervention strategies as well. Tilahun et al. [32] investigated a Pneumonia-Typhoid co-infection model with cost-effective optimal control analysis, incorporating preventive and treatment controls for both diseases. They showed that the strategy that combines Pneumonia treatment and Typhoid fever prevention is the most cost-effective in reducing the burden of the co-infections of Pnemonia and Typhoid fever. The researchers in [9] analyzed an optimal control model for HIV and TB co-infection, capturing resistance of HIV to anti-retroviral therapy. The authors showed that a combination of TB treatment and anti-retroviral therapy for HIV is the most effective in reducing the burden of the co-infection of the two diseases. In addition, Tanvi and Aggarwal [31] studied an HIV-TB co-infection, incorporating detection and treatment for both diseases. They observed that the strategy that implements detection for both diseases yielded the most economic and epidemic gains infighting the co-infections of the two diseases.

To the best of the authors' knowledge, optimal control analysis has never been applied to the coinfection of Human papillomavirus and TB in the literature. Hence, it will be imperative to investigate the optimal control and cost-effectiveness analysis of the co-infections of oncogenic HPV and TB. In particular, this paper extends the work of Omame et al. [24] by assessing the impact of HPV prevention and TB treatment controls on the control and management of the co-infections of the two diseases.

The rest of the paper is organized as follows. The model is formulated in Section 2 , alongside the basic properties of the model. The co-infection model without controls is analyzed qualitatively in Section 3 . The optimal control model is considered in Section 4. Simulations of the model are carried out in Section 5 while Section 6 gives the concluding remarks.

\section{Model formulation and basic properties of the model}

The total sexually active population at time $t$, denoted by $N_{\mathrm{H}}(t)$, is divided into eleven mutually-exclusive compartments: Susceptible individuals $(S(t))$, individuals infected with HPV $\left(I_{\mathrm{HP}}(t)\right)$, individuals who have recovered from or cleared HPV infection $\left(R_{\mathrm{HP}}(t)\right)$, individuals with persistent HPV infection $\left(P_{\mathrm{HP}}(t)\right)$, individuals with latent TB $\left(E_{\mathrm{T}}(t)\right)$, individuals with active TB infection $\left(A_{\mathrm{T}}(t)\right)$, individuals treated of TB $\left(T_{\mathrm{T}}(t)\right)$, individuals dually infected with HPV and latent TB $\left(I_{\mathrm{HE}}^{\mathrm{P}}(t)\right)$, individuals dually infected with HPV and active TB $I_{\mathrm{HA}}^{\mathrm{P}}$, individuals dually infected with persistent HPV and latent TB, $\left(P_{\mathrm{HE}}^{\mathrm{P}}(t)\right)$ individuals dually infected with persistent HPV and active $\left(P_{\mathrm{HA}}^{\mathrm{P}}(t)\right)$. Thus

$$
N_{\mathrm{H}}=S+I_{\mathrm{HP}}+R_{\mathrm{HP}}+P_{\mathrm{HP}}+E_{\mathrm{T}}+A_{\mathrm{T}}+T_{\mathrm{T}}+I_{\mathrm{HE}}^{\mathrm{P}}+I_{\mathrm{HA}}^{\mathrm{P}}+P_{\mathrm{HE}}^{\mathrm{P}}+P_{\mathrm{HA}}^{\mathrm{P}}
$$


medRxiv preprint doi: https://doi.org/10.1101/2020.09.15.20195297; this version posted September 27, 2020. The copyright holder for this preprint (which was not certified by peer review) is the author/funder, who has granted medRxiv a license to display the preprint in

It is made available under a CC-BY-NC-ND 4.0 International license .

Based on the above formulations and assumptions, the HPV-TB Coinfection model is given by the following deterministic system of non-linear differential equations (flow diagram of the model is shown in Figure 1, the associated state variables and parameters are well described in Tables 1 and 2):

$$
\begin{aligned}
\frac{d S}{d t} & =\Lambda_{\mathrm{H}}-\left(\lambda_{\mathrm{HP}}+\mu_{\mathrm{H}}+\lambda_{\mathrm{T}}\right) S \\
\frac{d I_{\mathrm{HP}}}{d t} & =\lambda_{\mathrm{HP}} S+r_{2}^{\mathrm{T}} I_{\mathrm{HA}}^{\mathrm{P}}+\lambda_{\mathrm{HP}} T_{\mathrm{T}}+\varphi_{\mathrm{P}} \lambda_{\mathrm{HP}} R_{\mathrm{HP}}-\left(\phi_{1}^{\mathrm{P}}+\lambda_{\mathrm{T}}+\mu_{\mathrm{H}}+\delta_{\mathrm{HP}}\right) I_{\mathrm{HP}} \\
\frac{d P_{\mathrm{HP}}}{d t} & =\left(1-\theta_{1}^{\mathrm{P}}\right) \phi_{1}^{\mathrm{P}} I_{\mathrm{HP}}+r_{3}^{\mathrm{T}} P_{\mathrm{HA}}^{\mathrm{P}}-\left(\rho_{1}^{\mathrm{P}}+\mu_{\mathrm{H}}+\lambda_{\mathrm{T}}\right) P_{\mathrm{HP}} \\
\frac{d R_{\mathrm{HP}}}{d t} & =\theta_{1}^{\mathrm{p}} \phi_{1}^{\mathrm{p}} I_{\mathrm{HP}}+\rho_{1}^{\mathrm{p}} P_{\mathrm{HP}}-\left(\varphi_{\mathrm{P}} \lambda_{\mathrm{HP}}+\mu_{\mathrm{H}}+\lambda_{\mathrm{T}}\right) R_{\mathrm{HP}} \\
\frac{d E_{\mathrm{T}}}{d t} & =\left(1-\xi_{1}^{\mathrm{T}}\right) \lambda_{\mathrm{T}}\left(S+R_{\mathrm{HP}}+\sigma_{\mathrm{T}} T_{\mathrm{T}}\right)+\theta_{2}^{\mathrm{P}} \phi_{2}^{\mathrm{P}} I_{\mathrm{HE}}^{\mathrm{P}}+\rho_{2}^{\mathrm{p}} P_{\mathrm{HE}}^{\mathrm{P}}-\left(\varepsilon_{1}^{\mathrm{T}} \lambda_{\mathrm{T}}+\gamma_{1}^{\mathrm{T}}+\varrho_{1}^{\mathrm{P}} \lambda_{\mathrm{HP}}+\mu_{\mathrm{H}}\right) E_{\mathrm{T}} \\
\frac{d A_{\mathrm{T}}}{d t} & =\xi_{1}^{\mathrm{T}} \lambda_{\mathrm{T}}\left(S+R_{\mathrm{HP}}+\sigma_{\mathrm{T}} T_{\mathrm{T}}\right)+\varepsilon_{1}^{\mathrm{T}} \lambda_{\mathrm{T}} E_{\mathrm{T}}+\gamma_{1}^{\mathrm{T}} E_{\mathrm{T}}+\theta_{3}^{\mathrm{P}} \phi_{3}^{\mathrm{P}} I_{\mathrm{HA}}^{\mathrm{P}}+\rho_{3}^{\mathrm{P}} P_{\mathrm{HA}}^{\mathrm{P}}-\left(r_{1}^{\mathrm{T}}+\mu_{\mathrm{H}}+\delta_{\mathrm{T}}+\varrho_{2}^{\mathrm{P}} \lambda_{\mathrm{HP}}\right) A_{\mathrm{T}} \\
\frac{d T_{\mathrm{T}}}{d t} & =r_{1}^{\mathrm{T}} A_{\mathrm{T}}-\left(\sigma_{\mathrm{T}} \lambda_{\mathrm{T}}+\lambda_{\mathrm{HP}}+\mu_{\mathrm{H}}\right) T_{\mathrm{T}} \\
\frac{d I_{\mathrm{HE}}^{\mathrm{P}}}{d t} & =\left(1-\xi_{2}^{\mathrm{T}}\right) \lambda_{\mathrm{T}} I_{\mathrm{HP}}+\varrho_{1}^{\mathrm{P}} \lambda_{\mathrm{HP}} E_{\mathrm{T}}-\left(\varepsilon_{2}^{\mathrm{T}} \lambda_{\mathrm{T}}+\gamma_{2}^{\mathrm{T}}+\mu_{\mathrm{H}}+\phi_{2}^{\mathrm{P}}+\delta_{\mathrm{HP} 1}\right) I_{\mathrm{HE}}^{\mathrm{P}} \\
\frac{d I_{\mathrm{HA}}^{\mathrm{P}}}{d t} & =\xi_{2}^{\mathrm{T}} \lambda_{\mathrm{T}} I_{\mathrm{HP}}+\varepsilon_{2}^{\mathrm{T}} \lambda_{\mathrm{T}} I_{\mathrm{HE}}^{\mathrm{P}}+\gamma_{2}^{\mathrm{T}} I_{\mathrm{HE}}^{\mathrm{P}}+\varrho_{2}^{\mathrm{P}} \lambda_{\mathrm{HP}} A_{\mathrm{T}}-\left(r_{2}^{\mathrm{T}}+\mu_{\mathrm{H}}+\delta_{\mathrm{T} 1}+\delta_{\mathrm{HP} 2}+\phi_{3}^{\mathrm{P}}\right) I_{\mathrm{HA}}^{\mathrm{P}} \\
\frac{d P_{\mathrm{HE}}^{\mathrm{P}}}{d t} & =\left(1-\xi_{3}^{\mathrm{T}}\right) \lambda_{\mathrm{T}} P_{\mathrm{HP}}+\left(1-\theta_{2}^{\mathrm{P}}\right) \phi_{2}^{\mathrm{P}} I_{\mathrm{HE}}^{\mathrm{P}}-\left(\varepsilon_{3}^{\mathrm{T}} \lambda_{\mathrm{T}}+\gamma_{3}^{\mathrm{T}}+\rho_{3}^{\mathrm{P}}+\mu_{\mathrm{H}}\right) P_{\mathrm{HE}}^{\mathrm{P}} \\
\frac{d P_{\mathrm{HA}}^{\mathrm{P}}}{d t} & =\xi_{3}^{\mathrm{T}} \lambda_{\mathrm{T}} P_{\mathrm{HP}}+\varepsilon_{3}^{\mathrm{T}} \lambda_{\mathrm{T}} P_{\mathrm{HE}}^{\mathrm{P}}+\gamma_{3}^{\mathrm{T}} P_{\mathrm{HE}}^{\mathrm{P}}+\left(1-\theta_{3}^{\mathrm{P}}\right) \phi_{3}^{\mathrm{P}} I_{\mathrm{HA}}^{\mathrm{P}}-\left(r_{3}^{\mathrm{T}}+\delta_{\mathrm{T} 2}+\rho_{3}^{\mathrm{P}}+\mu_{\mathrm{H}}\right) P_{\mathrm{HA}}^{\mathrm{P}}
\end{aligned}
$$

where:

$$
\begin{gathered}
\lambda_{\mathrm{HP}}=\frac{\beta_{\mathrm{HP}}\left[I_{\mathrm{HP}}+\eta_{p} P_{\mathrm{HP}}+\kappa_{\mathrm{T}}\left(I_{\mathrm{HA}}^{\mathrm{P}}+\eta_{\mathrm{T}} I_{\mathrm{HE}}^{\mathrm{P}}+\eta_{p} P_{\mathrm{HA}}^{\mathrm{P}}+\eta_{\mathrm{T}} \eta_{p} P_{\mathrm{HE}}^{\mathrm{P}}\right)\right]}{N_{\mathrm{H}}}, \\
\lambda_{\mathrm{T}}=\frac{\beta_{\mathrm{T}}\left[A_{\mathrm{T}}+\omega_{p}\left(I_{\mathrm{HA}}^{\mathrm{P}}+\eta_{p} P_{\mathrm{HA}}^{\mathrm{P}}\right)\right]}{N_{\mathrm{H}}}
\end{gathered}
$$

The parameters $\kappa_{\mathrm{T}}\left(\kappa_{\mathrm{T}} \geq 1\right)$ is a modification parameter accounting for the increased infectiousness of dually infected individuals due to tuberculosis, $\eta_{\mathrm{T}}\left(\eta_{\mathrm{T}} \leq 1\right)$ is a modification parameter accounting for reduced infectiousness of dually infected individuals due to latent TB. $\omega_{p}\left(\omega_{p} \geq 1\right)$ is a modification parameter accounting for the increased infectiousness of dually infected individuals due to HPV while $\eta_{p}\left(\eta_{p} \leq 1\right)$ is a modification parameter accounting for the reduced infectiousness of singly infected and dually infected individuals due to persistent HPV infection. This population is further reduced by natural death (at a rate $\mu_{\mathrm{H}}$ natural death occurs in all epidemiological compartments at this rate). In (2), $\beta_{\text {нг is }}$ the effective contact rate for transmission of HPV infection. 
medRxiv preprint doi: https://doi.org/10.1101/2020.09.15.20195297; this version posted September 27, 2020. The copyright holder for this preprint (which was not certified by peer review) is the author/funder, who has granted medRxiv a license to display the preprint in

It is made available under a CC-BY-NC-ND 4.0 International license .

Table 1: Description of variables in the model (1).

\begin{tabular}{ll}
\hline Variable & Interpretation \\
\hline$S$ & population of susceptible individuals \\
$I_{\mathrm{HP}}$ & Population of individuals infected with HPV \\
$R_{\mathrm{HP}}$ & Population of individuals who have recovered from HPV \\
$P_{\mathrm{HP}}$ & Population of individuals with persistent HPV infection \\
$E_{\mathrm{T}}$ & Population of individuals with latent TB \\
$A_{\mathrm{T}}$ & Population of individuals with active TB \\
$T_{\mathrm{T}}$ & Population of individuals treated of TB \\
$I_{\mathrm{HE}}^{\mathrm{P}}$ & Population of individuals dually infected with HPV and latent TB \\
$I_{\mathrm{HA}}^{\mathrm{P}}$ & Population of individuals dually infected with HPV and active TB \\
$P_{\mathrm{H}}^{\mathrm{P}}$ & Population of individuals dually infected with persistent HPV and latent TB \\
$P_{\mathrm{HA}}^{\mathrm{P}}$ & Population of individuals dually infected with persistent HPV and active TB \\
$N_{\mathrm{H}}$ & Total human population \\
\hline
\end{tabular}


medRxiv preprint doi: https://doi.org/10.1101/2020.09.15.20195297; this version posted September 27, 2020. The copyright holder for this preprint (which was not certified by peer review) is the author/funder, who has granted medRxiv a license to display the preprint in It is made available under a CC-BY-NC-ND 4.0 International license .

Table 2: Description of parameters in the model (1).

\begin{tabular}{|c|c|c|c|}
\hline Parameter & Interpretation & Value & References \\
\hline$\Lambda_{\mathrm{H}}$ & Recruitment rate & 164490 & 8 \\
\hline$\mu_{\mathrm{H}}$ & Natural death rate & 0.0128 & 8 \\
\hline$\phi_{i}^{\mathrm{P}} \quad i=1,2,3$ & $\begin{array}{l}\text { Recovery rates from HPV for individuals in the } I_{\mathrm{HP}}, I_{\mathrm{HE}}^{\mathrm{P}}, I_{\mathrm{HA}}^{\mathrm{P}} \text { classes, } \\
\text { respectively }\end{array}$ & 0.9 & 16 \\
\hline$i=1,2,3$ & Recovery rates from TB for individuals in the $A_{\mathrm{T}}, I_{\mathrm{HA}}^{\mathrm{P}}, P_{\mathrm{HA}}^{\mathrm{P}}$ classes & 2.0 & 24 \\
\hline$\theta_{i}^{\mathrm{P}} \quad i=1,2,3$ & $\begin{array}{l}\text { Fraction of infected individuals who recovered naturally from HPV (but did not develop } \\
\text { persistent HPV infection) }\end{array}$ & 0.5 & 16 \\
\hline$\rho_{i, i=1,2,3}^{\mathrm{P}}$ & Rate at which individuals recover naturally from the persistent infection & 0.895 & 24 \\
\hline$\varphi_{\mathrm{P}}$ & Rate of reinfection with HPV & 0.2 & 25 \\
\hline$\delta_{\mathrm{HP}}$ & Disease induced death rates for individuals singly infected with HPV & 0.001 & [16, 25] \\
\hline$\delta_{\mathrm{T}}$ & Disease induced death rates for individuals singly infected with tuberculosis & 0.07 & 21 \\
\hline$\delta_{\mathrm{T} 1}, \delta_{\mathrm{T} 2}$ & $\begin{array}{l}\text { TB-induced death rates for individuals dually infected with } \\
\text { active tuberculosis and HPV }\end{array}$ & 0.007 & 21 \\
\hline$\delta_{\mathrm{HP} 1}, \delta_{\mathrm{HP} 2}$ & HPV-induced death rates for mixed infections & 0.001 & 24 \\
\hline$\sigma_{\mathrm{T}}$ & Rate of reinfection with TB & 0.2 & 24 \\
\hline$i=1,2,3$ & Fraction of newly infected individuals with active TB & 0.7 & 24 \\
\hline$\varepsilon_{1}^{\mathrm{T}}$ & Exogenous re-infection rates to active TB & 1.5 & 22 \\
\hline $\begin{array}{l}\varepsilon_{i}^{\mathrm{T}} \\
\gamma_{1}^{\mathrm{T}}\end{array}$ & $\begin{array}{l}\text { Exogenous re-infection rates to active TB for individuals with mixed infections } \\
\text { Slow progression rate to active TB }\end{array}$ & $\begin{array}{l}1.5 \\
0.005\end{array}$ & Assumed \\
\hline $\begin{array}{ll}\gamma_{i}^{\mathrm{P}} & i=2,3 \\
\beta_{\mathrm{HP}} & \end{array}$ & $\begin{array}{l}\text { Slow progression rate to active TB for individuals with mixed infections } \\
\text { Effective contact rate for HPV transmission } \\
\text { Effective contact rate for TB transmission }\end{array}$ & $\begin{array}{l}0.005 \\
1.0 \\
8.557\end{array}$ & $\begin{array}{l}\text { Assumed } \\
{[16}\end{array}$ \\
\hline $\begin{array}{l}\beta_{\mathrm{T}} \\
\eta_{\mathrm{T}}\end{array}$ & $\begin{array}{l}\text { Effective contact rate for TB transmission } \\
\text { Modification parameter for reduced infectiousness of dually infected individuals with }\end{array}$ & 8.557 & 22 \\
\hline$\omega_{p}$ & $\begin{array}{l}\text { latent TB relative to those with active TB } \\
\text { Modification parameter for increased infectiousness of dually infected individuals due } \\
\text { to HPV }\end{array}$ & 0.9 & Assumed \\
\hline$\eta_{p}$ & $\begin{array}{l}\text { Modification parameter for reduced infectiousness of infected individuals with persistent } \\
\text { HPV relative to those infected with HPV }\end{array}$ & 0.9 & 23 \\
\hline$\varrho_{1}^{\mathrm{P}}$ & $\begin{array}{l}\text { Modification parameter accounting for increased susceptibility to HPV } \\
\text { by TB infected individuals }\end{array}$ & 1.3 & 39 \\
\hline$\varrho_{2}^{\mathrm{P}}$ & $\begin{array}{l}\text { Modification parameter accounting for increased susceptibility to HPV } \\
\text { by TB infected individuals with mixed infections }\end{array}$ & 1.3 & 39 \\
\hline
\end{tabular}


medRxiv preprint doi: https://doi.org/10.1101/2020.09.15.20195297; this version posted September 27, 2020. The copyright holder for this preprint (which was not certified by peer review) is the author/funder, who has granted medRxiv a license to display the preprint in It is made available under a CC-BY-NC-ND 4.0 International license .

\subsection{Basic properties of the co-infection model (1) without controls}

The basic qualitative properties of the oncogenic HPV-TB co-infection model (1) will now be considered. Specifically, we establish the following results.

\subsubsection{Positivity and boundedness of solutions}

For the oncogenic HPV-TB co-infection model (1) to be meaningful in the concept of epidemiological sense, it is necessary to prove that all its state variables are non- negative over time. The approach adopted by Omame et al [23] will be used to establish the results below.

Theorem 2.1 Let the initial data be

$S(0)>0, I_{\mathrm{HP}}(0) \geq 0, R_{\mathrm{HP}}(0) \geq 0, P_{\mathrm{HP}}(0) \geq 0, E_{\mathrm{T}}(0) \geq 0, A_{\mathrm{T}}(0) \geq 0, T_{\mathrm{T}}(0) \geq 0, I_{\mathrm{HE}}^{\mathrm{P}}(0) \geq 0, I_{\mathrm{HA}}^{\mathrm{P}}(0) \geq$ $0, P_{\mathrm{HE}}^{\mathrm{P}}(0) \geq 0, P_{\mathrm{HA}}^{\mathrm{P}}(0) \geq 0$

Then the solutions

$\left(S, I_{\mathrm{HP}}, R_{\mathrm{HP}}, P_{\mathrm{HP}}, E_{\mathrm{T}}, A_{\mathrm{T}}, T_{\mathrm{T}}, I_{\mathrm{HE}}^{\mathrm{P}}, I_{\mathrm{HA}}^{\mathrm{P}}, P_{\mathrm{HE}}^{\mathrm{P}}, P_{\mathrm{HA}}^{\mathrm{P}}\right)$ of the model (1) are non-negative for all time $t>0$.

Lemma 2.1 The region $\mathcal{D} \subset \mathfrak{R}_{+}^{11}$ is positively-invariant for the co-infection model (1) with initial conditions in $\mathfrak{R}_{+}^{11}$.

\section{Mathematical Analysis of the model without controls}

\subsection{Basic reproduction number of the co-infection model}

The co-infection model (1) has a DFE, obtained by setting the right-hand sides of the equations in the model (1) to zero, given by

$$
\begin{aligned}
\xi_{0} & =\left(S^{*}, I_{\mathrm{HP}}^{*}, P_{\mathrm{HP}}^{*}, R_{\mathrm{HP}}^{*}, E_{\mathrm{T}}^{*}, A_{\mathrm{T}}^{*}, T_{\mathrm{T}}^{*}, I_{\mathrm{HE}}^{\mathrm{p}^{*}}, I_{\mathrm{HA}}^{\mathrm{P}^{*}}, P_{\mathrm{HE}}^{\mathrm{P}^{*}}, P_{\mathrm{HA}}^{\mathrm{P}^{*}}\right) \\
& =\left(\frac{\Lambda_{\mathrm{H}}}{\mu_{\mathrm{H}}}, 0,0,0,0,0,0,0,0,0\right)
\end{aligned}
$$

The basic reproduction number of the oncogenic HPV-TB co-infection model (1), using the approach in van den Driessche and Watmough [35], is given by $\mathcal{R}_{0}=\max \left\{\mathcal{R}_{0 \mathrm{H}}, \mathcal{R}_{0 \mathrm{~T}}\right\}$ where $\mathcal{R}_{0 \mathrm{H}}$ and $\mathcal{R}_{0 \mathrm{~T}}$ are, respectively, the oncogenic HPV and TB associated reproduction numbers, given by

$$
\mathcal{R}_{0 \mathrm{H}}=\frac{\beta_{\mathrm{HP}}\left(\Delta_{2}+\eta_{\mathrm{P}} \varpi_{1}\right)}{\Delta_{1} \Delta_{2}}
$$

and

$$
\mathcal{R}_{0 \mathrm{~T}}=\frac{\beta_{\mathrm{T}}\left[\gamma_{1}^{\mathrm{T}}\left(1-\xi_{1}^{\mathrm{T}}\right)+\xi_{1}^{\mathrm{T}} \Delta_{3}\right]}{\Delta_{3} \Delta_{4}}
$$


medRxiv preprint doi: https://doi.org/10.1101/2020.09.15.20195297; this version posted September $27,2020$. The copyright holder for this preprint (which was not certified by peer review) is the author/funder, who has granted medRxiv a license to display the preprint in

It is made available under a CC-BY-NC-ND 4.0 International license.

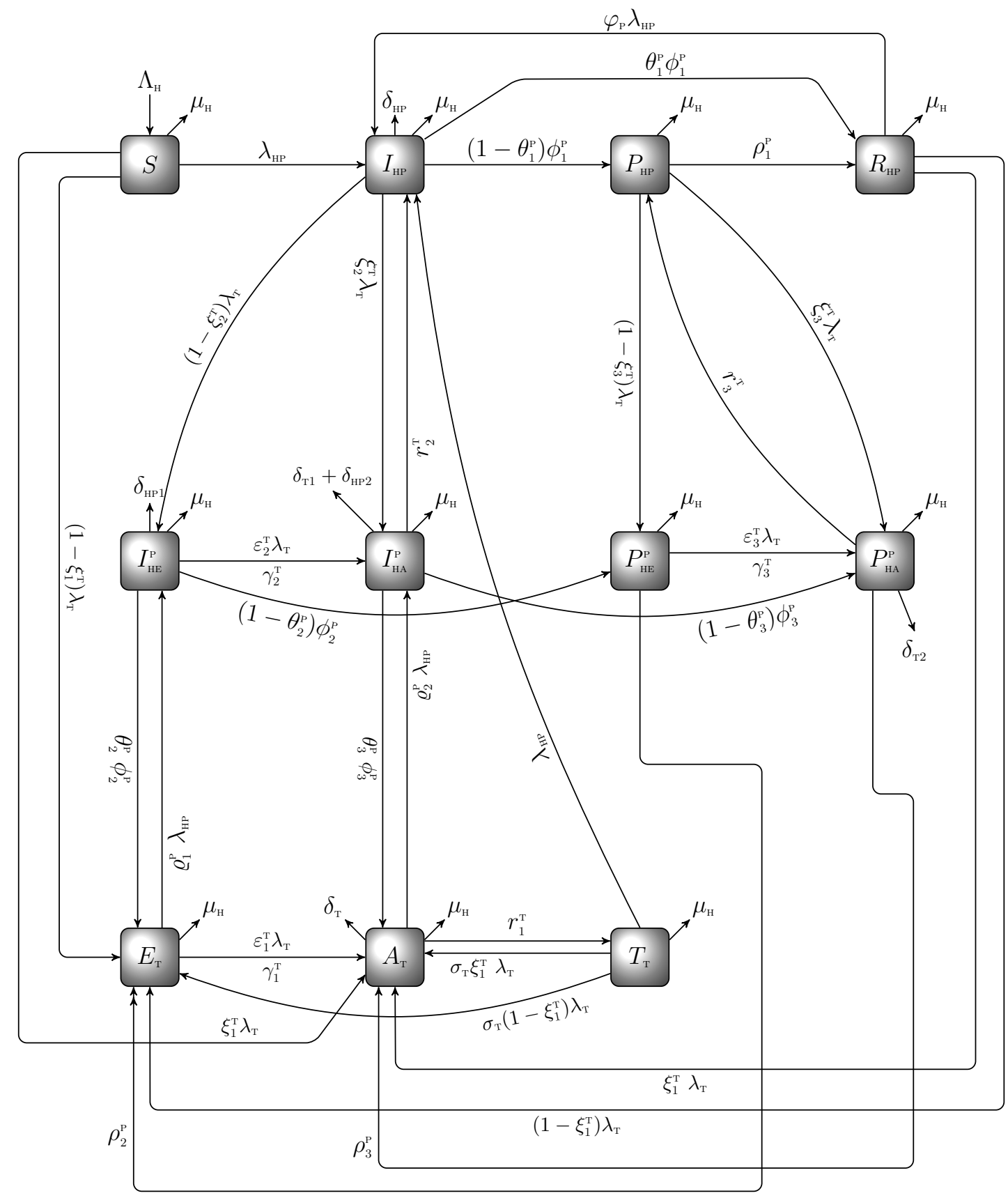

Figure 1: Schematic diagram of the model (1) 
medRxiv preprint doi: https://doi.org/10.1101/2020.09.15.20195297; this version posted September 27, 2020. The copyright holder for this preprint (which was not certified by peer review) is the author/funder, who has granted medRxiv a license to display the preprint in It is made available under a CC-BY-NC-ND 4.0 International license .

where,

$$
\Delta_{1}=\phi_{1}^{\mathrm{P}}+\delta_{\mathrm{HP}}+\mu_{\mathrm{H}}, \quad \Delta_{2}=\rho_{1}^{\mathrm{P}}+\mu_{\mathrm{H}}, \quad \Delta_{3}=\gamma_{1}^{\mathrm{T}}+\mu_{\mathrm{H}}, \quad \Delta_{4}=r_{1}^{\mathrm{T}}+\delta_{\mathrm{T}}+\mu_{\mathrm{H}}, \quad \varpi_{1}=\left(1-\theta_{1}^{\mathrm{P}}\right) \phi_{1}^{\mathrm{P}}
$$

Using Theorem 2 of [35], the following result is established.

Lemma 3.1 The DFE, $\xi_{0}$, of the oncogenic HPV-TB co-infection model (1) is locally asymptotically stable if $\mathcal{R}_{0}<1$, and unstable if $\mathcal{R}_{0}>1$.

\subsection{Global asymptotic stability(GAS) of the disease-free equilibrium(DFE) $\xi_{0}$ of the co-infection model}

We shall apply the approach illustrated in [5] to investigate the global asymptotic stability of the disease free equilibrium of the co-infection model. In this section, we list two conditions that if met, also guarantee the global asymptotic stability of the disease-free state. First, system (1) must be written in the form:

$$
\begin{aligned}
\frac{d V}{d t} & =P(V, K) \\
\frac{d K}{d t} & =Q(V, K), Q(V, 0)=0
\end{aligned}
$$

where $V \in R^{m}$ denotes (its components) the number of uninfected individuals and $K \in R^{n}$ denotes (its components) the number of infected individuals. $U_{0}=\left(V^{*}, 0\right)$ denotes the disease-free equilibrium of this system. The conditions $(W 1)$ and $(W 2)$ following must be satisfied in order to guarantee local asymptotic stability:

$(W 1)$ : For $\frac{d V}{d t}=P(V, 0), V^{*}$ is globally asymptotically stable (GAS),

$(W 2): Q(V, K)=B K-\hat{Q}(V, K) V, Q(V, K) \geq 0$ for $(V, K) \in \Omega$,

where $B=D_{K} Q\left(V^{*}, 0\right)$ is an M-matrix (the off-diagonal elements of $B$ are nonnegative) and $\Omega$ is the region where the model makes biological sense. If System (1) satisfies the above two conditions then the following theorem holds:

Theorem 3.1 The fixed point $U_{0}=\left(V^{*}, 0\right)$ is a globally asymptotic stable (GAS) equilibrium of (1) provided that $R_{0}<1$ (LAS) and that assumptions $(W 1)$ and $(W 2)$ are met

\section{Proof}

$$
\frac{d V}{d t}=P(V, K)=\left(\begin{array}{c}
\Lambda_{\mathrm{H}}-\left(\lambda_{\mathrm{HP}}+\mu_{\mathrm{H}}+\lambda_{\mathrm{T}}\right) S \\
\theta_{1}^{\mathrm{P}} \phi_{1}^{\mathrm{P}} I_{\mathrm{HP}}+\rho_{1}^{\mathrm{P}} P_{\mathrm{HP}}-\left(\varphi_{\mathrm{P}} \lambda_{\mathrm{HP}}+\mu_{\mathrm{H}}+\lambda_{\mathrm{T}}\right) R_{\mathrm{HP}} \\
r_{1}^{\mathrm{T}} A_{\mathrm{T}}-\left(\sigma_{\mathrm{T}} \lambda_{\mathrm{T}}+\lambda_{\mathrm{HP}}+\mu_{\mathrm{H}}\right) T_{\mathrm{T}}
\end{array}\right),
$$


medRxiv preprint doi: https://doi.org/10.1101/2020.09.15.20195297; this version posted September 27, 2020. The copyright holder for this preprint (which was not certified by peer review) is the author/funder, who has granted medRxiv a license to display the preprint in

$$
P(V, 0)=\left(\begin{array}{c}
\Lambda_{\mathrm{H}}-\mu_{\mathrm{H}} S \\
0 \\
0
\end{array}\right)
$$

where $V$ denotes the number of non-infectious compartments and $K$ denotes the number of infectious compartments

$$
Q(V, K)=\left(\begin{array}{c}
\lambda_{\mathrm{HP}} S+r_{2}^{\mathrm{T}} I_{\mathrm{HA}}^{\mathrm{P}}+\lambda_{\mathrm{HP}} T_{\mathrm{T}}+\varphi_{\mathrm{P}} \lambda_{\mathrm{HP}} R_{\mathrm{HP}}-\left(\phi_{1}^{\mathrm{P}}+\lambda_{\mathrm{T}}+\mu_{\mathrm{H}}+\delta_{\mathrm{HP}}\right) I_{\mathrm{HP}} \\
\left(1-\theta_{1}^{\mathrm{P}}\right) \phi_{1}^{\mathrm{P}} I_{\mathrm{HP}}+r_{3}^{\mathrm{T}} P_{\mathrm{HA}}^{\mathrm{P}}-\left(\rho_{1}^{\mathrm{P}}+\mu_{\mathrm{H}}+\lambda_{\mathrm{T}}\right) P_{\mathrm{HP}} \\
\left(1-\xi_{1}^{\mathrm{T}}\right) \lambda_{\mathrm{T}}\left(S+R_{\mathrm{HP}}+\sigma_{\mathrm{T}} T_{\mathrm{T}}\right)+\theta_{2}^{\mathrm{P}} \phi_{2}^{\mathrm{P}} I_{\mathrm{HE}}^{\mathrm{P}}+\rho_{2}^{\mathrm{p}} P_{\mathrm{HE}}^{\mathrm{P}}-\left(\varepsilon_{1}^{\mathrm{T}} \lambda_{\mathrm{T}}+\gamma_{1}^{\mathrm{T}}+\varrho_{1}^{\mathrm{p}} \lambda_{\mathrm{HP}}+\mu_{\mathrm{H}}\right) E_{\mathrm{T}} \\
\xi_{1}^{\mathrm{T}} \lambda_{\mathrm{T}}\left(S+R_{\mathrm{HP}}+\sigma_{\mathrm{T}} T_{\mathrm{T}}\right)+\varepsilon_{1}^{\mathrm{T}} \lambda_{\mathrm{T}} E_{\mathrm{T}}+\gamma_{1}^{\mathrm{T}} E_{\mathrm{T}}+\theta_{3}^{\mathrm{P}} \phi_{3}^{\mathrm{P}} I_{\mathrm{HA}}^{\mathrm{P}}+\rho_{3}^{\mathrm{p}} P_{\mathrm{HA}}^{\mathrm{P}}-\left(r_{1}^{\mathrm{T}}+\mu_{\mathrm{H}}+\delta_{\mathrm{T}}+\varrho_{2}^{\mathrm{p}} \lambda_{\mathrm{HP}}\right) A_{\mathrm{T}} \\
\left(1-\xi_{2}^{\mathrm{T}}\right) \lambda_{\mathrm{T}} I_{\mathrm{HP}}+\varrho_{1}^{\mathrm{p}} \lambda_{\mathrm{HP}} E_{\mathrm{T}}-\left(\varepsilon_{2}^{\mathrm{T}} \lambda_{\mathrm{T}}+\gamma_{2}^{\mathrm{T}}+\mu_{\mathrm{H}}+\phi_{2}^{\mathrm{p}}+\delta_{\mathrm{HP} 1}\right) I_{\mathrm{HE}}^{\mathrm{P}} \\
\xi_{2}^{\mathrm{T}} \lambda_{\mathrm{T}} I_{\mathrm{HP}}+\varepsilon_{2}^{\mathrm{T}} \lambda_{\mathrm{T}} I_{\mathrm{HE}}^{\mathrm{P}}+\gamma_{2}^{\mathrm{T}} I_{\mathrm{HE}}^{\mathrm{P}}+\varrho_{2}^{\mathrm{P}} \lambda_{\mathrm{HP}} A_{\mathrm{T}}-\left(r_{2}^{\mathrm{T}}+\mu_{\mathrm{H}}+\delta_{\mathrm{T} 1}+\delta_{\mathrm{HP} 2}+\phi_{3}^{\mathrm{P}}\right) I_{\mathrm{HA}}^{\mathrm{P}} \\
\left(1-\xi_{3}^{\mathrm{T}}\right) \lambda_{\mathrm{T}} P_{\mathrm{HP}}+\left(1-\theta_{2}^{\mathrm{P}}\right) \phi_{2}^{\mathrm{P}} I_{\mathrm{HE}}^{\mathrm{P}}-\left(\varepsilon_{3}^{\mathrm{T}} \lambda_{\mathrm{T}}+\gamma_{3}^{\mathrm{T}}+\rho_{3}^{\mathrm{P}}+\mu_{\mathrm{H}}\right) P_{\mathrm{HE}}^{\mathrm{P}} \\
\xi_{3}^{\mathrm{T}} \lambda_{\mathrm{T}} P_{\mathrm{HP}}+\varepsilon_{3}^{\mathrm{T}} \lambda_{\mathrm{T}} P_{\mathrm{HE}}^{\mathrm{P}}+\gamma_{3}^{\mathrm{T}} P_{\mathrm{HE}}^{\mathrm{P}}+\left(1-\theta_{3}^{\mathrm{P}}\right) \phi_{3}^{\mathrm{P}} I_{\mathrm{HA}}^{\mathrm{P}}-\left(r_{3}^{\mathrm{T}}+\delta_{\mathrm{T} 2}+\rho_{3}^{\mathrm{P}}+\mu_{\mathrm{H}}\right) P_{\mathrm{HA}}^{\mathrm{P}}
\end{array}\right),
$$

$B=D_{K} Q\left(V^{*}, 0\right)=\left(\begin{array}{cccccccc}\beta_{\mathrm{HP}}-\Delta_{1} & \beta_{\mathrm{HP}} \eta_{\mathrm{P}} & 0 & 0 & \beta_{\mathrm{HP}} \kappa_{\mathrm{T}} \eta_{\mathrm{T}} & \beta_{\mathrm{HP}} \kappa_{\mathrm{T}}+r_{2}^{\mathrm{T}} & \beta_{\mathrm{HP}} \kappa_{\mathrm{T}} \eta_{\mathrm{T}} \eta_{\mathrm{P}} & \beta_{\mathrm{HP}} \kappa_{\mathrm{T}} \eta_{\mathrm{T}} \\ \varpi_{1} & -\Delta_{2} & 0 & 0 & 0 & 0 & 0 & r_{3}^{\mathrm{T}} \\ 0 & 0 & -\Delta_{3} & \left(1-\xi_{1}^{\mathrm{T}}\right) \beta_{\mathrm{T}} & \theta_{2}^{\mathrm{P}} \phi_{2}^{\mathrm{P}} & \left(1-\xi_{1}^{\mathrm{T}}\right) \beta_{\mathrm{T}} \omega_{\mathrm{P}} & \rho_{2}^{\mathrm{P}} & \left(1-\xi_{1}^{\mathrm{T}}\right) \beta_{\mathrm{T}} \omega_{\mathrm{P}} \eta_{\mathrm{P}} \\ 0 & 0 & \gamma_{1}^{\mathrm{P}} & \xi_{1}^{\mathrm{T}} \beta_{\mathrm{T}}-\Delta_{4} & 0 & \xi_{1}^{\mathrm{T}} \beta_{\mathrm{T}} \omega_{\mathrm{P}}+\theta_{3}^{\mathrm{P}} \phi_{3}^{\mathrm{P}} & 0 & \xi_{1}^{\mathrm{T}} \beta_{\mathrm{T}} \omega_{\mathrm{P}} \eta_{\mathrm{P}}+\rho_{3}^{\mathrm{P}} \\ 0 & 0 & 0 & 0 & -\Delta_{5} & 0 & 0 & 0 \\ 0 & 0 & 0 & 0 & \gamma_{2}^{\mathrm{T}} & -\Delta_{6} & 0 & 0 \\ 0 & 0 & 0 & 0 & \varpi_{2} & 0 & -\Delta_{7} & 0 \\ 0 & 0 & 0 & 0 & 0 & \varpi_{3} & \gamma_{3}^{\mathrm{T}} & -\Delta_{8}\end{array}\right)$

$$
\hat{Q}(V, K)=\left(\begin{array}{c}
\beta_{\mathrm{HP}}\left[I_{\mathrm{HP}}+\eta_{\mathrm{P}} P_{\mathrm{HP}}+\kappa_{\mathrm{T}}\left(I_{\mathrm{HA}}^{\mathrm{P}}+\eta_{\mathrm{T}} I_{\mathrm{HE}}^{\mathrm{P}}+\eta_{\mathrm{P}} P_{\mathrm{HA}}^{\mathrm{P}}+\eta_{\mathrm{T}} \eta_{\mathrm{P}} P_{\mathrm{HE}}^{\mathrm{P}}\right)\right]\left(1-\frac{S+T}{N}\right)-\varphi_{\mathrm{P}} \lambda_{\mathrm{HP}} R_{\mathrm{HP}}+\lambda_{\mathrm{T}} I_{\mathrm{HP}} \\
\lambda_{\mathrm{T}} P_{\mathrm{HP}} \\
\left(1-\xi_{1}^{\mathrm{T}}\right) \beta_{\mathrm{T}}\left[A_{\mathrm{T}}+\omega_{p}\left(I_{\mathrm{HA}}^{\mathrm{P}}+\eta_{p} P_{\mathrm{HA}}^{\mathrm{P}}\right)\right]\left(1-\frac{S+R_{\mathrm{HP}}}{N}\right)-\left(1-\xi_{1}^{\mathrm{T}}\right) \lambda_{\mathrm{T}} \sigma_{\mathrm{T}} T_{\mathrm{T}}+\varepsilon_{1}^{\mathrm{T}} \lambda_{\mathrm{T}} E_{\mathrm{T}}+\varrho_{1}^{\mathrm{P}} \lambda_{\mathrm{HP}} E_{\mathrm{T}} \\
\xi_{1}^{\mathrm{T}} \beta_{\mathrm{T}}\left[A_{\mathrm{T}}+\omega_{p}\left(I_{\mathrm{HA}}^{\mathrm{P}}+\eta_{p} P_{\mathrm{HA}}^{\mathrm{P}}\right)\right]\left(1-\frac{S+R_{\mathrm{HP}}}{N}\right)-\xi_{1}^{\mathrm{T}} \lambda_{\mathrm{T}} \sigma_{\mathrm{T}} T_{\mathrm{T}}-\varepsilon_{1}^{\mathrm{T}} \lambda_{\mathrm{T}} E_{\mathrm{T}}+\varrho_{2}^{\mathrm{P}} \lambda_{\mathrm{HP}} A_{\mathrm{T}} \\
-\left(1-\xi_{2}^{\mathrm{T}}\right) \lambda_{\mathrm{T}} I_{\mathrm{HP}}-\varrho_{1}^{\mathrm{P}} \lambda_{\mathrm{HP}} E_{\mathrm{T}}+\varepsilon_{2}^{\mathrm{T}} \lambda_{\mathrm{T}} I_{\mathrm{HE}}^{\mathrm{P}} \\
-\xi_{2}^{\mathrm{T}} \lambda_{\mathrm{T}} I_{\mathrm{HP}}-\varrho_{2}^{\mathrm{P}} \lambda_{\mathrm{HP}} A_{\mathrm{T}}-\varepsilon_{2}^{\mathrm{T}} \lambda_{\mathrm{T}} I_{\mathrm{HE}}^{\mathrm{P}} \\
-\left(1-\xi_{3}^{\mathrm{T}}\right) \lambda_{\mathrm{T}} P_{\mathrm{HP}}+\varepsilon_{3}^{\mathrm{T}} \lambda_{\mathrm{T}} P_{\mathrm{HE}}^{\mathrm{P}} \\
-\xi_{3}^{\mathrm{T}} \lambda_{\mathrm{T}} P_{\mathrm{HP}}-\varepsilon_{3}^{\mathrm{T}} \lambda_{\mathrm{T}} P_{\mathrm{HE}}^{\mathrm{P}}
\end{array}\right.
$$

It is clear from the above, that, $\hat{Q}(V, K) \ngtr 0$. Hence the DFE may not be globally asymptotically stable, suggesting the possibility of a backward bifurcation. This supports the backward bifurcation analysis in the proceeding section. 
medRxiv preprint doi: https://doi.org/10.1101/2020.09.15.20195297; this version posted September 27, 2020. The copyright holder for this preprint (which was not certified by peer review) is the author/funder, who has granted medRxiv a license to display the preprint in

It is made available under a CC-BY-NC-ND 4.0 International license.

\subsection{Backward bifurcation analysis of the model without controls}

We shall carry out analysis in this section to know the type of bifurcation the model (1) may undergo, using the Centre Manifold Theory as illustrated in [6]. The following result can be established.

Theorem 3.2 Suppose a backward bifurcation coefficient $a>0$, (with a defined below), when $\mathcal{R}_{0}<1$

$$
\begin{aligned}
a & =-\frac{2 \beta_{\mathrm{HP}}^{*}}{N_{\mathrm{H}}^{*}}\left(\omega_{2}+\eta_{\mathrm{P}} \omega_{3}\right)\left\{\left(\omega_{2}+\omega_{3}+\omega_{4}+\omega_{5}+\omega_{6}\right) \nu_{2}+\left(\nu_{5}+\nu_{8}\right) \varrho_{1}^{\mathrm{P}} \omega_{5}+\left(\nu_{6}+\nu_{9}\right) \varrho_{2}^{\mathrm{P}} \omega_{6}-\varphi_{\mathrm{p}} \omega_{4}\right\} \\
& -\frac{2 \beta_{\mathrm{T}} \nu_{5}\left(1-\xi_{1}^{\mathrm{T}}\right) \omega_{6}}{N_{\mathrm{H}}^{*}}\left(\omega_{2}+\omega_{3}+\omega_{5}+\omega_{6}+\omega_{7}+\varepsilon_{1}^{\mathrm{T}} \omega_{5}-\sigma_{\mathrm{T}} \omega_{7}\right)-\frac{2 \beta_{\mathrm{T}} \nu_{6} \xi_{1}^{\mathrm{T}} \omega_{6}}{N_{\mathrm{H}}^{*}}\left(\omega_{2}+\omega_{3}+\omega_{5}+\omega_{6}+\omega_{7}-\varepsilon_{1}^{\mathrm{T}} \omega_{5}-\sigma_{\mathrm{T}} \omega_{7}\right) \\
& -\frac{2 \beta_{\mathrm{T}} \omega_{6}}{N_{\mathrm{H}}^{*}}\left\{\nu_{2} \omega_{2}+\nu_{3} \omega_{3}+\left[\left(1-\xi_{2}^{\mathrm{T}}\right) \nu_{8}-\xi_{1}^{\mathrm{T}} \nu_{9}\right] \omega_{2}+\left[\left(1-\xi_{3}^{\mathrm{T}}\right) \nu_{10}-\xi_{3}^{\mathrm{T}} \nu_{11}\right] \omega_{3}\right\}
\end{aligned}
$$

then model (1) exhibits backward bifurcation at $\mathcal{R}_{0}=1$. If $a<0$, then the system (1) exhibits a forward bifurcation at $\mathcal{R}_{0}=1$.

\section{Proof}

Suppose

$$
\xi_{e}=\left(S^{* *}, I_{\mathrm{HP}}^{* *}, P_{\mathrm{HP}}^{* *}, R_{\mathrm{HP}}^{* *}, E_{\mathrm{T}}^{* *}, A_{\mathrm{T}}^{* *}, T_{\mathrm{T}}^{* *}, I_{\mathrm{HE}}^{\mathrm{P} * *}, I_{\mathrm{HA}}^{\mathrm{P} * *}, P_{\mathrm{HE}}^{\mathrm{P} * *}, P_{\mathrm{HA}}^{\mathrm{p} *}\right)
$$

represents any arbitrary endemic equilibrium of the model. The existence of backward bifurcation will be studied using the Centre Manifold Theory [6]. To apply this theory, it is appropriate to do the following change of variables.

Let

$$
S=x_{1}, I_{\mathrm{HP}}=x_{2}, P_{\mathrm{HP}}=x_{3}, R_{\mathrm{HP}}=x_{4}, E_{\mathrm{T}}=x_{5}, A_{\mathrm{T}}=x_{6}, T_{\mathrm{T}}=x_{7}, I_{\mathrm{HE}}=x_{8}, I_{\mathrm{HA}}=x_{9}, P_{\mathrm{HE}}=x_{10}, P_{\mathrm{HA}}=x_{11}
$$

so that

$$
N_{\mathrm{H}}=\sum_{i=1}^{11} x_{i}
$$

Further, using the vector notation

$$
X=\left(x_{1}, x_{2}, x_{3}, x_{4}, x_{5}, x_{6}, x_{7}, x_{8}, x_{9}, x_{10}, x_{11}\right)^{T}
$$

the model (1) can be re-written in the form

$$
\frac{d X}{d t}=f=\left(f_{1}, f_{2}, f_{3}, f_{4}, f_{5}, f_{6}, f_{7}, f_{8}, f_{9}, f_{10}, f_{11}\right)^{T}
$$


medRxiv preprint doi: https://doi.org/10.1101/2020.09.15.20195297; this version posted September $27,2020$. The copyright holder for this preprint (which was not certified by peer review) is the author/funder, who has granted medRxiv a license to display the preprint in It is made available under a CC-BY-NC-ND 4.0 International license .

as follows:

$$
\begin{aligned}
\frac{d x_{1}}{d t} & =\Lambda_{\mathrm{H}}-\left(\lambda_{\mathrm{HP}}+\mu_{\mathrm{H}}+\lambda_{\mathrm{T}}\right) x_{1}:=f_{1} \\
\frac{d x_{2}}{d t} & =\lambda_{\mathrm{HP}} x_{1}+r_{2}^{\mathrm{T}} x_{9}+\lambda_{\mathrm{HP}} x_{7}+\varphi_{\mathrm{P}} \lambda_{\mathrm{HP}} x_{4}-\left(\phi_{1}^{\mathrm{P}}+\lambda_{\mathrm{T}}+\mu_{\mathrm{H}}+\delta_{\mathrm{HP}}\right) x_{2}:=f_{2} \\
\frac{d x_{3}}{d t} & =\left(1-\theta_{1}^{\mathrm{P}}\right) \phi_{1}^{\mathrm{P}} x_{2}+r_{3}^{\mathrm{T}} x_{11}-\left(\rho_{1}^{\mathrm{P}}+\mu_{\mathrm{H}}+\lambda_{\mathrm{T}}\right) x_{3}:=f_{3} \\
\frac{d x_{4}}{d t} & =\theta_{1}^{\mathrm{P}} \phi_{1}^{\mathrm{P}} x_{2}+\rho_{1}^{\mathrm{P}} x_{3}-\left(\varphi_{\mathrm{P}} \lambda_{\mathrm{HP}}+\mu_{\mathrm{H}}+\lambda_{\mathrm{T}}\right) x_{4}:=f_{4} \\
\frac{d x_{5}}{d t} & =\left(1-\xi_{1}^{\mathrm{T}}\right) \lambda_{\mathrm{T}}\left(x_{1}+x_{4}+\sigma_{\mathrm{T}} x_{7}\right)+\theta_{2}^{\mathrm{P}} \phi_{2}^{\mathrm{P}} x_{8}+\rho_{2}^{\mathrm{P}} x_{10}-\left(\varepsilon_{1}^{\mathrm{T}} \lambda_{\mathrm{T}}+\gamma_{1}^{\mathrm{T}}+\varrho_{1}^{\mathrm{P}} \lambda_{\mathrm{HP}}+\mu_{\mathrm{H}}\right) x_{5}:=f_{5} \\
\frac{d x_{6}}{d t} & =\xi_{1}^{\mathrm{T}} \lambda_{\mathrm{T}}\left(x_{1}+x_{4}+\sigma_{\mathrm{T}} x_{7}\right)+\varepsilon_{1}^{\mathrm{T}} \lambda_{\mathrm{T}} x_{5}+\gamma_{1}^{\mathrm{T}} x_{5}+\theta_{3}^{\mathrm{P}} \phi_{3}^{\mathrm{P}} x_{9}+\rho_{3}^{\mathrm{P}} x_{11}-\left(r_{1}^{\mathrm{T}}+\mu_{\mathrm{H}}+\delta_{\mathrm{T}}+\varrho_{2}^{\mathrm{P}} \lambda_{\mathrm{HP}}\right) x_{6}:=f_{6} \\
\frac{d x_{7}}{d t} & =r_{1}^{\mathrm{T}} x_{6}-\left(\sigma_{\mathrm{T}} \lambda_{\mathrm{T}}+\lambda_{\mathrm{HP}}+\mu_{\mathrm{H}}\right) x_{7}:=f_{7} \\
\frac{d x_{8}}{d t} & =\left(1-\xi_{2}^{\mathrm{T}}\right) \lambda_{\mathrm{T}} x_{2}+\varrho_{1}^{\mathrm{P}} \lambda_{\mathrm{HP}} x_{5}-\left(\varepsilon_{2}^{\mathrm{T}} \lambda_{\mathrm{T}}+\gamma_{2}^{\mathrm{T}}+\mu_{\mathrm{H}}+\phi_{2}^{\mathrm{P}}+\delta_{\mathrm{HP} 1}\right) x_{8}:=f_{8} \\
\frac{d x_{9}}{d t} & =\xi_{2}^{\mathrm{T}} \lambda_{\mathrm{T}} x_{2}+\varepsilon_{2}^{\mathrm{T}} \lambda_{\mathrm{T}} x_{8}+\gamma_{2}^{\mathrm{T}} x_{8}+\varrho_{2}^{\mathrm{P}} \lambda_{\mathrm{HP}} x_{6}-\left(r_{2}^{\mathrm{T}}+\mu_{\mathrm{H}}+\delta_{\mathrm{T} 1}+\delta_{\mathrm{HP} 2}+\phi_{3}^{\mathrm{P}}\right) x_{9}:=f_{9} \\
\frac{d x_{10}}{d t} & =\left(1-\xi_{3}^{\mathrm{T}}\right) \lambda_{\mathrm{T}} x_{3}+\left(1-\theta_{2}^{\mathrm{P}}\right) \phi_{2}^{\mathrm{P}} x_{8}-\left(\varepsilon_{3}^{\mathrm{T}} \lambda_{\mathrm{T}}+\gamma_{3}^{\mathrm{T}}+\rho_{3}^{\mathrm{P}}+\mu_{\mathrm{H}}\right) x_{10}:=f_{10} \\
\frac{d x_{11}}{d t} & =\xi_{3}^{\mathrm{T}} \lambda_{\mathrm{T}} x_{3}+\varepsilon_{3}^{\mathrm{T}} \lambda_{\mathrm{T}} x_{10}+\gamma_{3}^{\mathrm{T}} x_{10}+\left(1-\theta_{3}^{\mathrm{P}}\right) \phi_{3}^{\mathrm{P}} x_{9}-\left(r_{3}^{\mathrm{T}}+\delta_{\mathrm{T} 2}+\rho_{3}^{\mathrm{p}}+\mu_{\mathrm{H}}\right) x_{11}:=f_{11}
\end{aligned}
$$

with:

$$
\begin{gathered}
\lambda_{\mathrm{HP}}=\frac{\beta_{\mathrm{HP}}\left[x_{2}+\eta_{p} x_{3}+\kappa_{\mathrm{T}}\left(x_{9}+\eta_{\mathrm{T}} x_{8}+\eta_{p} x_{11}+\eta_{\mathrm{T}} \eta_{p} x_{10}\right)\right]}{\sum_{i=1}^{11} x_{i}}, \\
\lambda_{\mathrm{T}}=\frac{\beta_{\mathrm{T}}\left[x_{6}+\omega_{p}\left(x_{9}+\eta_{p} x_{11}\right)\right]}{\sum_{i=1}^{11} x_{i}}
\end{gathered}
$$

Consider the case when $\mathcal{R}_{0 \mathrm{H}}=1$. Assume, further, that $\beta_{\mathrm{HP}}$ is chosen as a bifurcation parameter. Solving for $\beta_{\mathrm{HP}}=\beta_{\mathrm{HP}}^{*}$ from $\mathcal{R}_{0 \mathrm{H}}=1$ gives

$$
\beta_{\mathrm{HP}}=\beta_{\mathrm{HP}}^{*}=\frac{\Delta_{1} \Delta_{2}}{\left(\Delta_{2}+\eta_{\mathrm{P}} \varpi_{1}\right)}
$$

Evaluating the Jacobian of the system (8) at the DFE, $J\left(\xi_{0}\right)$, and using the approach in [6], we have that $J\left(\xi_{0}\right)$ has a right eigenvector (associated with the simple zero eigenvalue) given by

$$
\mathbf{w}=\left[\omega_{1}, \omega_{2}, \omega_{3}, \omega_{4}, \omega_{5}, \omega_{6}, \omega_{7}, \omega_{8}, \omega_{9}, \omega_{10}, \omega_{11}\right]^{T}
$$


medRxiv preprint doi: https://doi.org/10.1101/2020.09.15.20195297; this version posted September 27, 2020. The copyright holder for this preprint (which was not certified by peer review) is the author/funder, who has granted medRxiv a license to display the preprint in

It is made available under a CC-BY-NC-ND 4.0 International license .

where,

$$
\begin{aligned}
& \omega_{1}=-\frac{1}{\mu_{\mathrm{H}}}\left(\beta_{\mathrm{HP}}^{*} \omega_{2}+\beta_{\mathrm{HP}}^{*} \eta_{\mathrm{P}} \omega_{3}+\beta_{\mathrm{T}}\right)<0, \\
& \omega_{2}=\omega_{2}>0, \\
& \omega_{3}=\frac{\varpi_{1} \omega_{2}}{\Delta_{2}}>0, \\
& \omega_{4}=\frac{\theta_{1}^{\mathrm{P}} \phi_{1}^{\mathrm{P}} \omega_{2}+\rho_{1}^{\mathrm{P}} \omega_{3}}{\mu_{\mathrm{H}}}>0, \\
& \omega_{5}=\frac{\left(1-\xi_{1}^{\mathrm{T}}\right) \beta_{\mathrm{T}} \omega_{6}}{\Delta_{3}}>0 \\
& \omega_{6}=\omega_{6}>0 \\
& \omega_{7}=\frac{r_{1}^{\mathrm{T}} \omega_{6}}{\mu_{\mathrm{H}}} \\
& \omega_{8}=\omega_{9}=\omega_{10}=\omega_{11}=0
\end{aligned}
$$

The components of the left eigenvector of $\left.J\left(\xi_{0}\right)\right|_{\beta_{\mathrm{S}}=\beta_{\mathrm{S}}^{*}}, \mathbf{v}=\left(\nu_{1}, \nu_{2}, \ldots, \nu_{11}\right)$, satisfying $\mathbf{v} \cdot \mathbf{w}=1$ are

$$
\begin{aligned}
\nu_{2} & =\nu_{2}>0 \\
\nu_{3} & =\frac{\beta_{\mathrm{HP}}^{*} \eta_{\mathrm{P}} \nu_{2}}{\Delta_{2}}>0 \\
\nu_{5} & =\frac{\gamma_{1}^{\mathrm{T}} \nu_{6}}{\Delta_{3}}>0 \\
\nu_{6} & =\nu_{6}>0 \\
\nu_{8} & =\frac{\beta_{\mathrm{HP}}^{*} \kappa_{\mathrm{T}} \eta_{\mathrm{T}} \nu_{2}+\theta_{2}^{\mathrm{P}} \phi_{2}^{\mathrm{P}} \nu_{5}+\gamma_{2}^{\mathrm{T}} \nu_{9}+\varpi_{2} \nu_{10}}{\Delta_{5}}>0 \\
\nu_{9} & =\frac{\left(\beta_{\mathrm{HP}}^{*} \kappa_{\mathrm{T}}+r_{2}^{\mathrm{T}}\right) \nu_{2}+\left(1-\xi_{1}^{\mathrm{T}}\right) \beta_{\mathrm{T}} \omega_{\mathrm{P}} \nu_{5}+\left(\xi_{1}^{\mathrm{T}} \beta_{\mathrm{T}} \omega_{\mathrm{P}}+\theta_{3}^{\mathrm{P}} \phi_{1}^{\mathrm{P}}\right) \nu_{6}+\varpi_{3} \nu_{11}}{\Delta_{6}}>0 \\
\nu_{10} & =\frac{\beta_{\mathrm{HP}}^{*} \kappa_{\mathrm{T}} \eta_{\mathrm{T}} \eta_{\mathrm{P}} \nu_{2}+\rho_{2}^{\mathrm{P}} \nu_{5}+\gamma_{3}^{\mathrm{T}} \nu_{11}}{\Delta_{7}}>0 \\
\nu_{11} & =\frac{\beta_{\mathrm{HP}}^{*} \kappa_{\mathrm{T}} \eta_{\mathrm{P}} \nu_{2}+r_{3}^{\mathrm{T}} \nu_{3}+\left(1-\xi_{1}^{\mathrm{T}}\right) \beta_{\mathrm{T}} \omega_{\mathrm{P}} \eta_{\mathrm{P}} \nu_{5}+\left(\xi_{1}^{\mathrm{T}} \beta_{\mathrm{T}} \omega_{\mathrm{P}} \eta_{\mathrm{P}}+\rho_{3}^{\mathrm{P}}\right) \nu_{6}}{\Delta_{6}}>0 \\
\nu_{1} & =\nu_{4}=\nu_{7}=0 .
\end{aligned}
$$

where,

$$
\begin{aligned}
& \Delta_{1}=\phi_{1}^{\mathrm{P}}+\delta_{\mathrm{HP}}+\mu_{\mathrm{H}}, \quad \Delta_{2}=\rho_{1}^{\mathrm{P}}+\mu_{\mathrm{H}}, \quad \Delta_{3}=\gamma_{1}^{\mathrm{T}}+\mu_{\mathrm{H}}, \quad \Delta_{4}=r_{1}^{\mathrm{T}}+\delta_{\mathrm{T}}+\mu_{\mathrm{H}}, \quad \Delta_{5}=\gamma_{2}^{\mathrm{T}}+\mu_{\mathrm{H}}+\phi_{2}^{\mathrm{P}}+\delta_{\mathrm{HP} 1}, \\
& \Delta_{6}=r_{2}^{\mathrm{T}}+\delta_{\mathrm{T} 1}+\delta_{\mathrm{HP} 2}+\phi_{3}^{\mathrm{P}}+\mu_{\mathrm{H}} \quad \Delta_{7}=\gamma_{3}^{\mathrm{T}}+\rho_{3}^{\mathrm{P}}+\mu_{\mathrm{H}}, \quad \Delta_{8}=r_{3}^{\mathrm{T}}+\delta_{\mathrm{T} 2}+\rho_{3}^{\mathrm{P}}+\mu_{\mathrm{H}}, \quad \varpi_{1}=\left(1-\theta_{1}^{\mathrm{P}}\right) \phi_{1}^{\mathrm{P}}, \\
& \varpi_{2}=\left(1-\theta_{2}^{\mathrm{P}}\right) \phi_{2}^{\mathrm{P}}, \quad \varpi_{3}=\left(1-\theta_{3}^{\mathrm{P}}\right) \phi_{3}^{\mathrm{P}}
\end{aligned}
$$

The associated bifurcation coefficients defined by $a$ and $b$, given by:

$$
a=\sum_{k, i, j=1}^{n} \nu_{k} \omega_{i} \omega_{j} \frac{\partial^{2} f_{k}}{\partial x_{i} \partial x_{j}}(0,0) \quad \text { and } \quad b=\sum_{k, i=1}^{n} \nu_{k} \omega_{i} \frac{\partial^{2} f_{k}}{\partial x_{i} \partial \beta_{S}^{*}}(0,0)
$$

are computed to be

$$
\begin{aligned}
a & =-\frac{2 \beta_{\mathrm{HP}}^{*}}{N_{\mathrm{H}}^{*}}\left(\omega_{2}+\eta_{\mathrm{P}} \omega_{3}\right)\left\{\left(\omega_{2}+\omega_{3}+\omega_{4}+\omega_{5}+\omega_{6}\right) \nu_{2}+\left(\nu_{5}+\nu_{8}\right) \varrho_{1}^{\mathrm{P}} \omega_{5}+\left(\nu_{6}+\nu_{9}\right) \varrho_{2}^{\mathrm{P}} \omega_{6}-\varphi_{\mathrm{P}} \omega_{4}\right\} \\
& -\frac{2 \beta_{\mathrm{T}} \nu_{5}\left(1-\xi_{1}^{\mathrm{T}}\right) \omega_{6}}{N_{\mathrm{H}}^{*}}\left(\omega_{2}+\omega_{3}+\omega_{5}+\omega_{6}+\omega_{7}+\varepsilon_{1}^{\mathrm{T}} \omega_{5}-\sigma_{\mathrm{T}} \omega_{7}\right)-\frac{2 \beta_{\mathrm{T}} \nu_{6} \xi_{1}^{\mathrm{T}} \omega_{6}}{N_{\mathrm{H}}^{*}}\left(\omega_{2}+\omega_{3}+\omega_{5}+\omega_{6}+\omega_{7}-\varepsilon_{1}^{\mathrm{T}} \omega_{5}-\sigma_{\mathrm{T}} \omega_{7}\right) \\
& -\frac{2 \beta_{\mathrm{T}} \omega_{6}}{N_{\mathrm{H}}^{*}}\left\{\nu_{2} \omega_{2}+\nu_{3} \omega_{3}+\left[\left(1-\xi_{2}^{\mathrm{T}}\right) \nu_{8}-\xi_{1}^{\mathrm{T}} \nu_{9}\right] \omega_{2}+\left[\left(1-\xi_{3}^{\mathrm{T}}\right) \nu_{10}-\xi_{3}^{\mathrm{T}} \nu_{11}\right] \omega_{3}\right\}
\end{aligned}
$$


and

$$
b=\sum_{k, i=1}^{20} \nu_{k} \omega_{i} \frac{\partial^{2} f_{k}}{\partial x_{i} \partial \beta^{*}}(0,0)=\left(\omega_{2}+\eta_{\mathrm{P}} \omega_{3}\right) \nu_{2}>0
$$

Since the bifurcation coefficient $b$ is positive, it follows from Theorem 4.1 in 6 ] that the model (1), or the transformed model (8), will undergo a backward bifurcation if the backward bifurcation coefficient, $a$, given by (11) is positive. Setting the HPV and TB re-infection paramters $\varphi_{\mathrm{P}}=0, \sigma_{\mathrm{T}}=0$ and the TB exogenous re-infection term $\varepsilon_{1}^{\mathrm{T}}=0$, the bifurcation coefficient, $a<0\left(\right.$ since $\left(1-\xi_{2}^{\mathrm{T}}\right) \nu_{8}>\xi_{2}^{\mathrm{T}} \nu_{9}>0$ and $\left(1-\xi_{3}^{\mathrm{T}}\right) \nu_{10}>\xi_{3}^{\mathrm{T}} \nu_{11}>0$, based on the definition of the components in 9), and also noting that all other components of the left and right eigenvectors occuring in the coefficient, $a$, are positive, including the parameters of the model. Hence, backward bifurcation does not occur in the Oncogenic HPV-TB co-infection model, in the absence of HPV and TB re-infection and in the absence of exogenous re-infection.

\section{Optimal control model}

In this section, we shall use the Pontryagin's Maximum Principle to determine the necessary conditions for the optimal control of the oncogenic HPV-TB co-infection model. We incorporate time dependent controls into the model (1) to determine the optimal strategy for curbing the co-infections of the two diseases. Thus, we have,

$$
\begin{aligned}
\frac{d S}{d t} & =\Lambda_{\mathrm{H}}-\left(\left(1-u_{1}\right) \lambda_{\mathrm{HP}}+\mu_{\mathrm{H}}+\lambda_{\mathrm{T}}\right) S \\
\frac{d I_{\mathrm{HP}}}{d t} & =\left(1-u_{1}\right) \lambda_{\mathrm{HP}} S+u_{3} r_{2}^{\mathrm{T}} I_{\mathrm{HA}}^{\mathrm{P}}+\lambda_{\mathrm{HP}} T_{\mathrm{T}}+\varphi_{\mathrm{P}} \lambda_{\mathrm{HP}} R_{\mathrm{HP}}-\left(\phi_{1}^{\mathrm{P}}+\lambda_{\mathrm{T}}+\mu_{\mathrm{H}}+\delta_{\mathrm{HP}}\right) I_{\mathrm{HP}} \\
\frac{d P_{\mathrm{HP}}}{d t} & =\left(1-\theta_{1}^{\mathrm{P}}\right) \phi_{1}^{\mathrm{P}} I_{\mathrm{HP}}+u_{3} r_{3}^{\mathrm{T}} P_{\mathrm{HA}}^{\mathrm{P}}-\left(\rho_{1}^{\mathrm{P}}+\mu_{\mathrm{H}}+\lambda_{\mathrm{T}}\right) P_{\mathrm{HP}} \\
\frac{d R_{\mathrm{HP}}}{d t} & =\theta_{1}^{\mathrm{P}} \phi_{1}^{\mathrm{P}} I_{\mathrm{HP}}+\rho_{1}^{\mathrm{P}} P_{\mathrm{HP}}-\left(\varphi_{\mathrm{P}} \lambda_{\mathrm{HP}}+\mu_{\mathrm{H}}+\lambda_{\mathrm{T}}\right) R_{\mathrm{HP}} \\
\frac{d E_{\mathrm{T}}}{d t} & =\left(1-\xi_{1}^{\mathrm{T}}\right) \lambda_{\mathrm{T}}\left(S+R_{\mathrm{HP}}+\sigma_{\mathrm{T}} T_{\mathrm{T}}\right)+\theta_{2}^{\mathrm{P}} \phi_{2}^{\mathrm{P}} I_{\mathrm{HE}}^{\mathrm{P}}+\rho_{2}^{\mathrm{P}} P_{\mathrm{HE}}^{\mathrm{P}}-\left(\varepsilon_{1}^{\mathrm{T}} \lambda_{\mathrm{T}}+\gamma_{1}^{\mathrm{T}}+\varrho_{1}^{\mathrm{P}}\left(1-u_{2}\right) \lambda_{\mathrm{HP}}+\mu_{\mathrm{H}}\right) E_{\mathrm{T}} \\
\frac{d A_{\mathrm{T}}}{d t} & =\xi_{1}^{\mathrm{T}} \lambda_{\mathrm{T}}\left(S+R_{\mathrm{HP}}+\sigma_{\mathrm{T}} T_{\mathrm{T}}\right)+\varepsilon_{1}^{\mathrm{T}} \lambda_{\mathrm{T}} E_{\mathrm{T}}+\gamma_{1}^{\mathrm{T}} E_{\mathrm{T}}+\theta_{3}^{\mathrm{P}} \phi_{3}^{\mathrm{P}} I_{\mathrm{HA}}^{\mathrm{P}}+\rho_{3}^{\mathrm{P}} P_{\mathrm{HA}}^{\mathrm{P}}-\left(r_{1}^{\mathrm{T}}+\mu_{\mathrm{H}}+\delta_{\mathrm{T}}+\varrho_{2}^{\mathrm{P}}\left(1-u_{2}\right) \lambda_{\mathrm{HP}}\right) A_{\mathrm{T}} \\
\frac{d T_{\mathrm{T}}}{d t} & =r_{1}^{\mathrm{T}} A_{\mathrm{T}}-\left(\sigma_{\mathrm{T}} \lambda_{\mathrm{T}}+\lambda_{\mathrm{HP}}+\mu_{\mathrm{H}}\right) T_{\mathrm{T}} \\
\frac{d I_{\mathrm{HE}}^{\mathrm{P}}}{d t} & =\left(1-\xi_{2}^{\mathrm{T}}\right) \lambda_{\mathrm{T}} I_{\mathrm{HP}}+\varrho_{1}^{\mathrm{P}}\left(1-u_{2}\right) \lambda_{\mathrm{HP}} E_{\mathrm{T}}-\left(\varepsilon_{2}^{\mathrm{T}} \lambda_{\mathrm{T}}+\gamma_{2}^{\mathrm{T}}+\mu_{\mathrm{H}}+\phi_{2}^{\mathrm{P}}+\delta_{\mathrm{HP} 1}\right) I_{\mathrm{HE}}^{\mathrm{P}} \\
\frac{d I_{\mathrm{HA}}^{\mathrm{P}}}{d t} & =\xi_{2}^{\mathrm{T}} \lambda_{\mathrm{T}} I_{\mathrm{HP}}+\varepsilon_{2}^{\mathrm{T}} \lambda_{\mathrm{T}} I_{\mathrm{HE}}^{\mathrm{P}}+\gamma_{2}^{\mathrm{T}} I_{\mathrm{HE}}^{\mathrm{P}}+\varrho_{2}^{\mathrm{P}}\left(1-u_{2}\right) \lambda_{\mathrm{HP}} A_{\mathrm{T}}-\left(u_{3} r_{2}^{\mathrm{T}}+\mu_{\mathrm{H}}+\delta_{\mathrm{T} 1}+\delta_{\mathrm{HP} 2}+\phi_{3}^{\mathrm{P}}\right) I_{\mathrm{HA}}^{\mathrm{P}} \\
\frac{d P_{\mathrm{HE}}^{\mathrm{P}}}{d t} & =\left(1-\xi_{3}^{\mathrm{T}}\right) \lambda_{\mathrm{T}} P_{\mathrm{HP}}+\left(1-\theta_{2}^{\mathrm{P}}\right) \phi_{2}^{\mathrm{P}} I_{\mathrm{HE}}^{\mathrm{P}}-\left(\varepsilon_{3}^{\mathrm{T}} \lambda_{\mathrm{T}}+\gamma_{3}^{\mathrm{T}}+\rho_{3}^{\mathrm{P}}+\mu_{\mathrm{H}}\right) P_{\mathrm{HE}}^{\mathrm{P}} \\
\frac{d P_{\mathrm{HA}}^{\mathrm{P}}}{d t} & =\xi_{3}^{\mathrm{T}} \lambda_{\mathrm{T}} P_{\mathrm{HP}}+\varepsilon_{3}^{\mathrm{T}} \lambda_{\mathrm{T}} P_{\mathrm{HE}}^{\mathrm{P}}+\gamma_{3}^{\mathrm{T}} P_{\mathrm{HE}}^{\mathrm{P}}+\left(1-\theta_{3}^{\mathrm{P}}\right) \phi_{3}^{\mathrm{P}} I_{\mathrm{HA}}^{\mathrm{P}}-\left(u_{3} r_{3}^{\mathrm{T}}+\delta_{\mathrm{T} 2}+\rho_{3}^{\mathrm{P}}+\mu_{\mathrm{H}}\right) P_{\mathrm{HA}}^{\mathrm{P}}
\end{aligned}
$$

subject to the initial conditions $S(0)=S^{0}, I_{\mathrm{HP}}(0)=I_{\mathrm{HP}}^{0}, P_{\mathrm{HP}}(0)=P_{\mathrm{HP}}^{0}, R_{\mathrm{HP}}(0)=R_{\mathrm{HP}}^{0}, E_{\mathrm{T}}(0)=E_{\mathrm{T}}^{0}, A_{\mathrm{T}}(0)=A_{\mathrm{T}}^{0}, T_{\mathrm{T}}(0)=$ $T_{\mathrm{T}}^{0}, I_{\mathrm{HE}}^{\mathrm{P}}(0)=I_{\mathrm{HE}}^{\mathrm{P} 0}, I_{\mathrm{HA}}^{\mathrm{P}}(0)=I_{\mathrm{HA}}^{\mathrm{P} 0}, P_{\mathrm{HE}}^{\mathrm{P}}(0)=P_{\mathrm{HE}}^{\mathrm{P} 0}, P_{\mathrm{HA}}^{\mathrm{P}}(0)=P_{\mathrm{HA}}^{\mathrm{P} 0}$

with:

$$
\begin{gathered}
\lambda_{\mathrm{HP}}=\frac{\beta_{\mathrm{HP}}\left[I_{\mathrm{HP}}+\eta_{p} P_{\mathrm{HP}}+\kappa_{\mathrm{T}}\left(I_{\mathrm{HA}}^{\mathrm{P}}+\eta_{\mathrm{T}} I_{\mathrm{HE}}^{\mathrm{P}}+\eta_{p} P_{\mathrm{HA}}^{\mathrm{P}}+\eta_{\mathrm{T}} \eta_{p} P_{\mathrm{HE}}^{\mathrm{P}}\right)\right]}{N_{\mathrm{H}}}, \\
\lambda_{\mathrm{T}}=\frac{\beta_{\mathrm{T}}\left[A_{\mathrm{T}}+\omega_{p}\left(I_{\mathrm{HA}}^{\mathrm{P}}+\eta_{p} P_{\mathrm{HA}}^{\mathrm{P}}\right)\right]}{N_{\mathrm{H}}}
\end{gathered}
$$

The control functions, $u_{1}(t), u_{2}(t)$, and $u_{3}(t)$ are bounded, Lebesgue integrable functions. The control $u_{1}(t)$ represents the efforts at preventing incident HPV infections. The control $u_{2}(t)$ aims at preventing infection with HPV by TB infected individuals so as to reduce the co-infection cases. TB Treatment control for individuals dually infected with oncogenic HPV and TB is denoted by $u_{3}(t)$. The controls $u_{1}$ and $u_{2}$ satisfies $0 \leq u_{1}, u_{2} \leq 1$ while the control $u_{3}$ satisfies $0<u_{3} \leq h$, where $h$ is the TB drug efficacy used for the treatment of co-infected individuals. Our optimal control problem involves a scenario where the number of HPV-infected, TB-infected, the co-infection cases and the cost of implementing preventive and treatment controls $u_{1}(t), u_{2}(t)$ and $u_{3}(t)$ are minimized subject to the state system (12). For this, we consider the 
medRxiv preprint doi: https://doi.org/10.1101/2020.09.15.20195297; this version posted September 27, 2020. The copyright holder for this preprint (which was not certified by peer review) is the author/funder, who has granted medRxiv a license to display the preprint in It is made available under a CC-BY-NC-ND 4.0 International license .

objective functional

$$
J\left[u_{1}, u_{2}, u_{3}\right]=\int_{0}^{T}\left[I_{\mathrm{HP}}(t)+P_{\mathrm{HP}}(t)+E_{\mathrm{T}}(t)+A_{\mathrm{T}}(t)+I_{\mathrm{HE}}(t)+I_{\mathrm{HA}}(t)+P_{\mathrm{HE}}(t)+P_{\mathrm{HA}}(t)+\frac{\chi_{1}}{2} u_{1}^{2}+\frac{\chi_{2}}{2} u_{2}^{2}+\frac{\chi_{3}}{2} u_{3}^{2}\right] d t
$$

$T$ is the final time. We seek to find an optimal control, $u_{1}^{*}, u_{2}^{*}, u_{3}^{*}$, such that

$$
J\left(u_{1}^{*}, u_{2}^{*}, u_{3}^{*}\right)=\min \left\{J\left(u_{1}^{*}, u_{2}^{*}, u_{3}^{*}\right) \mid u_{1}, u_{2}, u_{3} \in U\right\}
$$

where $U=\left\{\left(u_{1}^{*}, u_{2}^{*}, u_{3}^{*}\right)\right\}$ such that $u_{1}^{*}, u_{2}^{*}, u_{3}^{*}$ are measurable with $0 \leq u_{1}^{*} \leq 1,0 \leq u_{2}^{*} \leq 1,0 \leq u_{3}^{*} \leq r$, for $t \in[0, T]$ is the control set. We shall now establish the existence of such an optimal solution which minimizes the objective functional $J$.

Theorem 4.1 Given the objective functional $J$, defined on the control set $U$, and subject to the state system 12 with non-negative initial conditions at $t=0$, then there exists an optimal control triple $u^{*}=\left(u_{1}, u_{2}, u_{3}\right)$ such that $J\left(u^{*}\right)=$ $\min \left\{J\left(u_{1}, u_{2}, u_{3}\right) \mid u_{1}, u_{2}, u_{3} \in U\right\}$.

We shall prove the existence of the given optimal control $u^{*}$, along with the corresponding solution trajectories by following the approach used in Mohammed-Awel and Numfor [18.

Proof:

The state functions are positive and the controls are Lebesgue measurable, therefore we have that $J\left(u_{i}\right) \geq 0$ for all $u_{i} \in U$. As a result, $\inf _{u_{i} \in U} J\left(u_{i}\right)$ exists and is finite. Therefore, a minimizing sequnce of controls $\left(u_{i}\right) \in U$ exists such that

$$
\lim _{n \rightarrow \infty} J\left(u_{i}^{n}\right)=\inf _{u_{i} \in U} J\left(u_{i}\right)
$$

Let $S^{n}, I_{\mathrm{HP}}^{n}, P_{\mathrm{HP}}^{n}, R_{\mathrm{HP}}^{n}, E_{\mathrm{T}}^{n}, A_{\mathrm{T}}^{n}, T_{\mathrm{T}}^{n}, I_{\mathrm{HE}}^{p n}, I_{\mathrm{HA}}^{p n}, P_{\mathrm{HE}}^{p n}, P_{\mathrm{HA}}^{p n}$ be the associated state trajectories. Since the state sequences are uniformly bounded, we have that the derivatives are also uniformly bounded. As a result, the state sequences are Lipschitz continuous with the same constant. Applying the Arzela-Ascoli Theorem [13], there exist $S^{*}, I_{\mathrm{HP}}^{*}, P_{\mathrm{HP}}^{*}, R_{\mathrm{HP}}^{*}, E_{\mathrm{T}}^{*}, A_{\mathrm{T}}^{*}, T_{\mathrm{T}}^{*}, I_{\mathrm{HE}}^{\mathrm{P}}, I_{\mathrm{HA}}^{\mathrm{P} *}, P_{\mathrm{HE}}^{\mathrm{P}^{*}}, P_{\mathrm{HA}}^{\mathrm{P}^{*}}$, such that on a sub-sequence

$$
\left(S^{n}, I_{\mathrm{HP}}^{n}, P_{\mathrm{HP}}^{n}, R_{\mathrm{HP}}^{n}, E_{\mathrm{T}}^{n}, A_{\mathrm{T}}^{n}, T_{\mathrm{T}}^{n}, I_{\mathrm{HE}}^{p n}, I_{\mathrm{HA}}^{p n}, P_{\mathrm{HE}}^{p n}, P_{\mathrm{HA}}^{p n}\right) \rightarrow \xi^{*} \quad \text { uniformly on } \quad[0, T]
$$

where,

$$
\xi^{*}=\left(S^{*}, I_{\mathrm{HP}}^{*}, P_{\mathrm{HP}}^{*}, R_{\mathrm{HP}}^{*}, E_{\mathrm{T}}^{*}, A_{\mathrm{T}}^{*}, T_{\mathrm{T}}^{*}, I_{\mathrm{HE}}^{\mathrm{P}^{*}}, I_{\mathrm{HA}}^{\mathrm{P} *}, P_{\mathrm{HE}}^{\mathrm{P}^{*}}, P_{\mathrm{HA}}^{\mathrm{P}^{*}}\right)
$$

Since $\left\|u_{i}\right\|_{L^{\infty}}<K$ for some $K>0$, it follows that $u_{i} \in L^{2}([0, T])$, such that on a sub-sequence

$$
u_{i} \rightarrow u_{i}^{n} \quad \text { weakly in } \quad L^{2}([0, T]), n \rightarrow \infty
$$

Applying the lower semi-continuity of $L^{2}$ norm with respect to weak convergence, we have that

$$
\begin{aligned}
\inf _{u_{i} \in U} J\left(u_{i}\right) & =\lim _{n \rightarrow \infty} J\left(u_{i}^{n}\right) \\
& =\lim _{n \rightarrow \infty} \int_{0}^{T}\left(I_{\mathrm{HP}}^{n}(t)+P_{\mathrm{HP}}^{n}(t)+E_{\mathrm{T}}^{n}(t)+A_{\mathrm{T}}^{n}(t)+I_{\mathrm{HE}}^{p n}(t)+I_{\mathrm{HA}}^{p n}(t)+P_{\mathrm{HE}}^{p n}(t)+P_{\mathrm{HA}}^{p n}(t)+\frac{\chi_{1}}{2}\left(u_{1}^{n}\right)^{2}+\frac{\chi_{2}}{2}\left(u_{2}^{n}\right)^{2}+\frac{\chi_{3}}{2}\left(u_{3}^{n}\right)^{2}\right) d t \\
& \geq \lim _{n \rightarrow \infty} \int_{0}^{T}\left(I_{\mathrm{HP}}^{*}(t)+P_{\mathrm{HP}}^{*}(t)+E_{\mathrm{T}}^{*}(t)+A_{\mathrm{T}}^{*}(t)+I_{\mathrm{HE}}^{p *}(t)+I_{\mathrm{HA}}^{p *}(t)+P_{\mathrm{HE}}^{p *}(t)+P_{\mathrm{HA}}^{p *}(t)+\frac{\chi_{1}}{2}\left(u_{1}^{*}\right)^{2}+\frac{\chi_{2}}{2}\left(u_{2}^{*}\right)^{2}+\frac{\chi_{3}}{2}\left(u_{3}^{*}\right)^{2}\right) d t \\
& =J\left(u_{i}^{*}\right)
\end{aligned}
$$

Considering the convergence of the sequences

$$
\left\{S^{n}\right\}_{n=1}^{\infty},\left\{I_{\mathrm{HP}}^{n}\right\}_{n=1}^{\infty},\left\{P_{\mathrm{HP}}^{n}\right\}_{n=1}^{\infty},\left\{R_{\mathrm{HP}}^{n}\right\}_{n=1}^{\infty},\left\{E_{\mathrm{T}}^{n}\right\}_{n=1}^{\infty},\left\{A_{\mathrm{T}}^{n}\right\}_{n=1}^{\infty},\left\{T_{\mathrm{T}}^{n}\right\}_{n=1}^{\infty},\left\{I_{\mathrm{HE}}^{p n}\right\}_{n=1}^{\infty},\left\{I_{\mathrm{HA}}^{p n}\right\}_{n=1}^{\infty},\left\{P_{\mathrm{HE}}^{p n}\right\}_{n=1}^{\infty},\left\{P_{\mathrm{HA}}^{p n}\right\}_{n=1}^{\infty}
$$

and passing to the limit in the ordinary differential equation system 12 , we have that $S^{*}, I_{\mathrm{HP}}^{*}, P_{\mathrm{HP}}^{*}, R_{\mathrm{HP}}^{*}, E_{\mathrm{T}}^{*}, A_{\mathrm{T}}^{*}, T_{\mathrm{T}}^{*}, I_{\mathrm{HE}}^{\mathrm{P} *}, I_{\mathrm{HA}}^{\mathrm{P}}, P_{\mathrm{HE}}^{\mathrm{P}^{*}}, P_{\mathrm{HA}}^{\mathrm{P}^{*}}$ are the states corresponding to the control triple $\left(u_{i}\right)$. Hence, $\left(u_{i}\right)$ is an optimal control triple. 
medRxiv preprint doi: https://doi.org/10.1101/2020.09.15.20195297; this version posted September 27, 2020. The copyright holder for this preprint (which was not certified by peer review) is the author/funder, who has granted medRxiv a license to display the preprint in

It is made available under a CC-BY-NC-ND 4.0 International license .

The Pontryagin's Maximum Principle [28] gives the necessary conditions which an optimal control pair must satisfy. This principle transforms 12 , 13 and 14 into a problem of minimizing a Hamiltonian, $\mathcal{H}$, pointwisely with regards to the control functions, $u_{1}, u_{2}, u_{3}$ :

$$
\begin{aligned}
& \mathcal{H}=I_{\mathrm{HP}}(t)+P_{\mathrm{HP}}(t)+E_{\mathrm{T}}(t)+A_{\mathrm{T}}(t)+I_{\mathrm{HE}}(t)+I_{\mathrm{HA}}(t)+P_{\mathrm{HE}}(t)+P_{\mathrm{HA}}(t)+\frac{\chi_{1}}{2} u_{1}^{2}+\frac{\chi_{2}}{2} u_{2}^{2}+\frac{\chi_{3}}{2} u_{3}^{2} \\
& +\lambda_{S}\left[\Lambda_{\mathrm{H}}-\left(\left(1-u_{1}\right) \lambda_{\mathrm{HP}}+\mu_{\mathrm{H}}+\lambda_{\mathrm{T}}\right) S\right] \\
& +\lambda_{I_{H P}}\left[\left(1-u_{1}\right) \lambda_{\mathrm{HP}} S+u_{3} r_{2}^{\mathrm{T}} I_{\mathrm{HA}}^{\mathrm{P}}+\lambda_{\mathrm{HP}} T_{\mathrm{T}}+\varphi_{\mathrm{P}} \lambda_{\mathrm{HP}} R_{\mathrm{HP}}-\left(\phi_{1}^{\mathrm{P}}+\lambda_{\mathrm{T}}+\mu_{\mathrm{H}}+\delta_{\mathrm{HP}}\right) I_{\mathrm{HP}}\right] \\
& +\lambda_{P_{H P}}\left[\left(1-\theta_{1}^{\mathrm{P}}\right) \phi_{1}^{\mathrm{P}} I_{\mathrm{HP}}+u_{3} r_{3}^{\mathrm{T}} P_{\mathrm{HA}}^{\mathrm{P}}-\left(\rho_{1}^{\mathrm{P}}+\mu_{\mathrm{H}}+\lambda_{\mathrm{T}}\right) P_{\mathrm{HP}}\right] \\
& +\lambda_{R_{H P}}\left[\theta_{1}^{\mathrm{P}} \phi_{1}^{\mathrm{P}} I_{\mathrm{HP}}+\rho_{1}^{\mathrm{P}} P_{\mathrm{HP}}-\left(\varphi_{\mathrm{P}} \lambda_{\mathrm{HP}}+\mu_{\mathrm{H}}+\lambda_{\mathrm{T}}\right) R_{\mathrm{HP}}\right] \\
& +\lambda_{E_{T}}\left[\left(1-\xi_{1}^{\mathrm{T}}\right) \lambda_{\mathrm{T}}\left(S+R_{\mathrm{HP}}+\sigma_{\mathrm{T}} T_{\mathrm{T}}\right)+\theta_{2}^{\mathrm{P}} \phi_{2}^{\mathrm{P}} I_{\mathrm{HE}}^{\mathrm{P}}+\rho_{2}^{\mathrm{P}} P_{\mathrm{HE}}^{\mathrm{P}}-\left(\varepsilon_{1}^{\mathrm{T}} \lambda_{\mathrm{T}}+\gamma_{1}^{\mathrm{T}}+\varrho_{1}^{\mathrm{P}}\left(1-u_{2}\right) \lambda_{\mathrm{HP}}+\mu_{\mathrm{H}}\right) E_{\mathrm{T}}\right] \\
& +\lambda_{A_{T}}\left[\xi_{1}^{\mathrm{T}} \lambda_{\mathrm{T}}\left(S+R_{\mathrm{HP}}+\sigma_{\mathrm{T}} T_{\mathrm{T}}\right)+\varepsilon_{1}^{\mathrm{T}} \lambda_{\mathrm{T}} E_{\mathrm{T}}+\gamma_{1}^{\mathrm{T}} E_{\mathrm{T}}+\theta_{3}^{\mathrm{P}} \phi_{3}^{\mathrm{P}} I_{\mathrm{HA}}^{\mathrm{P}}+\rho_{3}^{\mathrm{P}} P_{\mathrm{HA}}^{\mathrm{P}}-\left(r_{1}^{\mathrm{T}}+\mu_{\mathrm{H}}+\delta_{\mathrm{T}}+\varrho_{2}^{\mathrm{P}}\left(1-u_{2}\right) \lambda_{\mathrm{HP}}\right) A_{\mathrm{T}}\right] \\
& +\lambda_{T_{T}}\left[r_{1}^{\mathrm{T}} A_{\mathrm{T}}-\left(\sigma_{\mathrm{T}} \lambda_{\mathrm{T}}+\lambda_{\mathrm{HP}}+\mu_{\mathrm{H}}\right) T_{\mathrm{T}}\right] \\
& +\lambda_{I_{H E}^{P}}\left[\left(1-\xi_{2}^{\mathrm{T}}\right) \lambda_{\mathrm{T}} I_{\mathrm{HP}}+\varrho_{1}^{\mathrm{P}}\left(1-u_{2}\right) \lambda_{\mathrm{HP}} E_{\mathrm{T}}-\left(\varepsilon_{2}^{\mathrm{T}} \lambda_{\mathrm{T}}+\gamma_{2}^{\mathrm{T}}+\mu_{\mathrm{H}}+\phi_{2}^{\mathrm{P}}+\delta_{\mathrm{HP} 1}\right) I_{\mathrm{HE}}^{\mathrm{P}}\right] \\
& +\lambda_{I_{H A}^{P}}\left[\xi_{2}^{\mathrm{T}} \lambda_{\mathrm{T}} I_{\mathrm{HP}}+\varepsilon_{2}^{\mathrm{T}} \lambda_{\mathrm{T}} I_{\mathrm{HE}}^{\mathrm{P}}+\gamma_{2}^{\mathrm{T}} I_{\mathrm{HE}}^{\mathrm{P}}+\varrho_{2}^{\mathrm{P}}\left(1-u_{2}\right) \lambda_{\mathrm{HP}} A_{\mathrm{T}}-\left(u_{3} r_{2}^{\mathrm{T}}+\mu_{\mathrm{H}}+\delta_{\mathrm{T} 1}+\delta_{\mathrm{HP} 2}+\phi_{3}^{\mathrm{P}}\right) I_{\mathrm{HA}}^{\mathrm{P}}\right] \\
& \left.+\lambda_{P_{H E}^{P}}\left[\xi_{3}^{\mathrm{T}}\right) \lambda_{\mathrm{T}} P_{\mathrm{HP}}+\left(1-\theta_{2}^{\mathrm{P}}\right) \phi_{2}^{\mathrm{P}} I_{\mathrm{HE}}^{\mathrm{P}}-\left(\varepsilon_{3}^{\mathrm{T}} \lambda_{\mathrm{T}}+\gamma_{3}^{\mathrm{T}}+\rho_{3}^{\mathrm{P}}+\mu_{\mathrm{H}}\right) P_{\mathrm{HE}}^{\mathrm{P}}\right] \\
& +\lambda_{P_{H A}^{P}}\left[\xi_{3}^{\mathrm{T}} \lambda_{\mathrm{T}} P_{\mathrm{HP}}+\varepsilon_{3}^{\mathrm{T}} \lambda_{\mathrm{T}} P_{\mathrm{HE}}^{\mathrm{P}}+\gamma_{3}^{\mathrm{T}} P_{\mathrm{HE}}^{\mathrm{P}}+\left(1-\theta_{3}^{\mathrm{P}}\right) \phi_{3}^{\mathrm{P}} I_{\mathrm{HA}}^{\mathrm{P}}-\left(u_{3} r_{3}^{\mathrm{T}}+\delta_{\mathrm{T} 2}+\rho_{3}^{\mathrm{P}}+\mu_{\mathrm{H}}\right) P_{\mathrm{HA}}^{\mathrm{P}}\right]
\end{aligned}
$$

Theorem 4.2 For an optimal control set $u_{1}, u_{2}, u_{3}$ that minimizes $J$ over $U$, there are adjoint variables, $\lambda_{1}, \lambda_{2}, \ldots, \lambda_{11}$ satisfying

$$
-\frac{\partial \lambda_{i}}{\partial t}=\frac{\partial \mathcal{H}}{\partial i}
$$

and with transversality conditions

$$
\lambda_{i}\left(t_{f}\right)=0, \quad \text { where, } \quad i=S, I_{\mathrm{HP}}, P_{\mathrm{HP}}, R_{\mathrm{HP}}, E_{\mathrm{T}}, A_{\mathrm{T}}, T_{\mathrm{T}}, I_{\mathrm{HE}}^{\mathrm{P}}, I_{\mathrm{HA}}^{\mathrm{P}}, P_{\mathrm{HE}}^{\mathrm{P}}, P_{\mathrm{HA}}^{\mathrm{P}} .
$$

Furthermore,

$$
\begin{aligned}
& u_{1}^{*}=\max \left\{0, \min \left(1, \frac{\left(\lambda_{2}-\lambda_{1}\right) \beta_{\mathrm{HP}} S\left[I_{\mathrm{HP}}+\eta_{p} P_{\mathrm{HP}}+\kappa_{\mathrm{T}}\left(I_{\mathrm{HA}}^{\mathrm{P}}+\eta_{\mathrm{T}} I_{\mathrm{HE}}^{\mathrm{P}}+\eta_{p} P_{\mathrm{HA}}^{\mathrm{P}}+\eta_{\mathrm{T}} \eta_{p} P_{\mathrm{HE}}^{\mathrm{P}}\right)\right]}{\chi_{1} N_{\mathrm{H}}}\right)\right\} \\
& u_{2}^{*}=\max \left\{0, \min \left(1, \frac{\left[\left(\lambda_{9}-\lambda_{6}\right) \varrho_{2}^{\mathrm{P}} A_{\mathrm{T}}+\left(\lambda_{8}-\lambda_{5}\right) \varrho_{1}^{\mathrm{P}} E_{\mathrm{T}}\right]\left[I_{\mathrm{HP}}+\eta_{p} P_{\mathrm{HP}}+\kappa_{\mathrm{T}}\left(I_{\mathrm{HA}}^{\mathrm{P}}+\eta_{\mathrm{T}} I_{\mathrm{HE}}^{\mathrm{P}}+\eta_{p} P_{\mathrm{HA}}^{\mathrm{P}}+\eta_{\mathrm{T}} \eta_{p} P_{\mathrm{HE}}^{\mathrm{P}}\right)\right]}{\chi_{2} N_{\mathrm{H}}}\right)\right\} \\
& u_{3}^{*}=\max \left\{0, \min \left(1, \frac{\left(\lambda_{9}-\lambda_{2}\right) I_{\mathrm{HA}} r_{2}+\left(\lambda_{11}-\lambda_{3}\right) P_{\mathrm{HA}} r_{3}}{\chi_{3}}\right)\right\}
\end{aligned}
$$

\section{Proof of Theorem 4.2}

Suppose $U^{*}=\left(u_{1}^{*}, u_{2}^{*}, u_{3}^{*}\right)$ is an optimal control and $S^{*}, I_{\mathrm{HP}}^{*}, P_{\mathrm{HP}}^{*}, P_{\mathrm{HP}}^{*}, R_{\mathrm{HP}}^{*}, E_{\mathrm{T}}^{*}, A_{\mathrm{T}}^{*}, T_{\mathrm{T}}^{*}, I_{\mathrm{HE}}^{\mathrm{P}^{*}}, I_{\mathrm{HA}}^{\mathrm{P}}, P_{\mathrm{HE}}^{\mathrm{P}^{*}}, P_{\mathrm{HA}}^{\mathrm{P}^{*}}$ are the corresponding state solutions. Applying the Pontryagin's Maximum Principle [28, there exist adjoint variables satisfying:

$$
\begin{aligned}
& -\frac{d \lambda_{S}}{d t}=\frac{\partial \mathcal{H}}{\partial S}, \quad \lambda_{\mathrm{S}}\left(t_{f}\right)=0, \quad-\frac{d \lambda_{I_{\mathrm{HP}}}}{d t}=\frac{\partial \mathcal{H}}{\partial I_{\mathrm{HP}}}, \quad \lambda_{I_{\mathrm{HP}}}\left(t_{f}\right)=0, \quad-\frac{d \lambda_{P_{\mathrm{HP}}}}{d t}=\frac{\partial \mathcal{H}}{\partial P_{\mathrm{HP}}}, \quad \lambda_{P_{\mathrm{HP}}}\left(t_{f}\right)=0, \\
& -\frac{d \lambda_{R_{\mathrm{HP}}}}{d t}=\frac{\partial \mathcal{H}}{\partial R_{\mathrm{HP}}}, \quad \lambda_{R_{\mathrm{HP}}}\left(t_{f}\right)=0,-\frac{d \lambda_{E_{\mathrm{T}}}}{d t}=\frac{\partial \mathcal{H}}{\partial E_{\mathrm{T}}}, \quad \lambda_{E_{\mathrm{T}}}\left(t_{f}\right)=0, \quad-\frac{d \lambda_{A_{\mathrm{T}}}}{d t}=\frac{\partial \mathcal{H}}{\partial A_{\mathrm{T}}}, \quad \lambda_{A_{\mathrm{T}}}\left(t_{f}\right)=0, \\
& -\frac{d \lambda_{T_{\mathrm{T}}}}{d t}=\frac{\partial \mathcal{H}}{\partial T_{\mathrm{T}}}, \quad \lambda_{T_{\mathrm{T}}}\left(t_{f}\right)=0, \quad-\frac{d \lambda_{I_{\mathrm{HE}}^{\mathrm{P}}}}{d t}=\frac{\partial \mathcal{H}}{\partial I_{\mathrm{HE}}^{\mathrm{P}}}, \quad \lambda_{I_{\mathrm{HE}}^{\mathrm{P}}}\left(t_{f}\right)=0, \quad-\frac{d \lambda_{I_{\mathrm{HA}}^{\mathrm{P}}}}{d t}=\frac{\partial \mathcal{H}}{\partial I_{\mathrm{HA}}^{\mathrm{P}}}, \quad \lambda_{I_{\mathrm{HA}}^{\mathrm{P}}}\left(t_{f}\right)=0 \\
& -\frac{d \lambda_{P_{\mathrm{HE}}^{\mathrm{P}}}}{d t}=\frac{\partial \mathcal{H}}{\partial P_{\mathrm{HE}}^{\mathrm{P}}}, \quad \lambda_{P_{\mathrm{HE}}^{\mathrm{P}}}\left(t_{f}\right)=0 \quad-\frac{d \lambda_{P_{\mathrm{HA}}^{\mathrm{P}}}}{d t}=\frac{\partial \mathcal{H}}{\partial P_{\mathrm{HA}}^{\mathrm{P}}}, \quad \lambda_{P_{\mathrm{HA}}^{\mathrm{P}}}\left(t_{f}\right)=0
\end{aligned}
$$

with transversality conditions;

$\lambda_{S}\left(t_{f}\right)=\lambda_{I_{\mathrm{HP}}}\left(t_{f}\right)=\lambda_{P_{\mathrm{HP}}}\left(t_{f}\right)=\lambda_{R_{\mathrm{HP}}}\left(t_{f}\right)=\lambda_{E_{\mathrm{T}}}\left(t_{f}\right)=\lambda_{A_{\mathrm{T}}}\left(t_{f}\right)=\lambda_{T_{\mathrm{T}}}\left(t_{f}\right)=\lambda_{I_{\mathrm{HE}}^{\mathrm{P}}}\left(t_{f}\right)=\lambda_{I_{\mathrm{HA}}^{\mathrm{P}}}\left(t_{f}\right)=\lambda_{P_{\mathrm{HE}}^{\mathrm{P}}}\left(t_{f}\right)=\lambda_{P_{\mathrm{HA}}^{\mathrm{P}}}\left(t_{f}\right)=0$

We can determine the behaviour of the control by differentiating the Hamiltonian, $\mathcal{H}$ with respect to the controls $\left(u_{1}, u_{2}, u_{3}\right)$ 
at $t$. On the interior of the control set, where $0<u_{j}<1$ for all $(j=1,2,3)$, we obtain

$$
\begin{aligned}
& 0=\frac{\partial \mathcal{H}}{\partial u_{1}}=\chi_{1} N_{\mathrm{H}} u_{1}^{*}-\left(\lambda_{2}-\lambda_{1}\right) \beta_{\mathrm{HP}} S\left[I_{\mathrm{HP}}+\eta_{p} P_{\mathrm{HP}}+\kappa_{\mathrm{T}}\left(I_{\mathrm{HA}}^{\mathrm{P}}+\eta_{\mathrm{T}} I_{\mathrm{HE}}^{\mathrm{P}}+\eta_{p} P_{\mathrm{HA}}^{\mathrm{P}}+\eta_{\mathrm{T}} \eta_{p} P_{\mathrm{HE}}^{\mathrm{P}}\right)\right] \\
& 0=\frac{\partial \mathcal{H}}{\partial u_{2}}=\chi_{2} N_{\mathrm{H}} u_{2}^{*}-\left[\left(\lambda_{9}-\lambda_{6}\right) \varrho_{2}^{\mathrm{P}} A_{\mathrm{T}}+\left(\lambda_{8}-\lambda_{5}\right) \varrho_{1}^{\mathrm{P}} E_{\mathrm{T}}\right]\left[I_{\mathrm{HP}}+\eta_{p} P_{\mathrm{HP}}+\kappa_{\mathrm{T}}\left(I_{\mathrm{HA}}^{\mathrm{P}}+\eta_{\mathrm{T}} I_{\mathrm{HE}}^{\mathrm{P}}+\eta_{p} P_{\mathrm{HA}}^{\mathrm{P}}+\eta_{\mathrm{T}} \eta_{p} P_{\mathrm{HE}}^{\mathrm{P}}\right)\right] \\
& 0=\frac{\partial \mathcal{H}}{\partial u_{3}}=\chi_{3} u_{3}^{*}-\left(\lambda_{9}-\lambda_{2}\right) I_{\mathrm{HA}} r_{2}-\left(\lambda_{11}-\lambda_{3}\right) P_{\mathrm{HA}} r_{3}
\end{aligned}
$$

Therefore, we have that 14 .

$$
\begin{gathered}
u_{1}^{*}=\frac{\left(\lambda_{2}-\lambda_{1}\right) \beta_{\mathrm{HP}} S\left[I_{\mathrm{HP}}+\eta_{p} P_{\mathrm{HP}}+\kappa_{\mathrm{T}}\left(I_{\mathrm{HA}}^{\mathrm{P}}+\eta_{\mathrm{T}} I_{\mathrm{HE}}^{\mathrm{P}}+\eta_{p} P_{\mathrm{HA}}^{\mathrm{P}}+\eta_{\mathrm{T}} \eta_{p} P_{\mathrm{HE}}^{\mathrm{P}}\right)\right]}{\chi_{1} N_{\mathrm{H}}}, \\
u_{2}^{*}=\frac{\left[\left(\lambda_{9}-\lambda_{6}\right) \varrho_{2}^{\mathrm{P}} A_{\mathrm{T}}+\left(\lambda_{8}-\lambda_{5}\right) \varrho_{1}^{\mathrm{P}} E_{\mathrm{T}}\right]\left[I_{\mathrm{HP}}+\eta_{p} P_{\mathrm{HP}}+\kappa_{\mathrm{T}}\left(I_{\mathrm{HA}}^{\mathrm{P}}+\eta_{\mathrm{T}} I_{\mathrm{HE}}^{\mathrm{P}}+\eta_{p} P_{\mathrm{HA}}^{\mathrm{P}}+\eta_{\mathrm{T}} \eta_{p} P_{\mathrm{HE}}^{\mathrm{P}}\right)\right]}{\chi_{2} N_{\mathrm{H}}}, \\
u_{3}^{*}=\frac{\left(\lambda_{9}-\lambda_{2}\right) I_{\mathrm{HA}} r_{2}+\left(\lambda_{11}-\lambda_{3}\right) P_{\mathrm{HA}} r_{3}}{\chi_{3}} \\
u_{1}^{*}=\max \left\{0, \min \left(1, \frac{\left(\lambda_{2}-\lambda_{1}\right) \beta_{\mathrm{HP}} S\left[I_{\mathrm{HP}}+\eta_{p} P_{\mathrm{HP}}+\kappa_{\mathrm{T}}\left(I_{\mathrm{HA}}^{\mathrm{P}}+\eta_{\mathrm{T}} I_{\mathrm{HE}}^{\mathrm{P}}+\eta_{p} P_{\mathrm{HA}}^{\mathrm{P}}+\eta_{\mathrm{T}} \eta_{p} P_{\mathrm{HE}}^{\mathrm{P}}\right)\right]}{\chi_{1} N_{\mathrm{H}}}\right)\right\}, \\
u_{2}^{*}=\max \left\{0, \min \left(1, \frac{\left[\left(\lambda_{9}-\lambda_{6}\right) \varrho_{2}^{\mathrm{P}} A_{\mathrm{T}}+\left(\lambda_{8}-\lambda_{5}\right) \varrho_{1}^{\mathrm{P}} E_{\mathrm{T}}\right]\left[I_{\mathrm{HP}}+\eta_{p} P_{\mathrm{HP}}+\kappa_{\mathrm{T}}\left(I_{\mathrm{HA}}^{\mathrm{P}}+\eta_{\mathrm{T}} I_{\mathrm{HE}}^{\mathrm{P}}+\eta_{p} P_{\mathrm{HA}}^{\mathrm{P}}+\eta_{\mathrm{T}} \eta_{p} P_{\mathrm{HE}}^{\mathrm{P}}\right)\right]}{\chi_{2} N_{\mathrm{H}}}\right)\right\}, \\
u_{3}^{*}=\max \left\{0, \min \left(1, \frac{\left(\lambda_{9}-\lambda_{2}\right) I_{\mathrm{HA}} r_{2}+\left(\lambda_{11}-\lambda_{3}\right) P_{\mathrm{HA}} r_{3}}{\chi_{3}}\right)\right\},
\end{gathered}
$$

\section{Simulations}

In this section, we shall explore uncertainty and sensitivity analyses on the parameters of the model due to lack of precision in the estimates of some of the co-infection model parameters and the uncertainty which may occur when gathering data for the simulations. We shall also carry out numerical simulations of the optimal control model (12), in order to assess the impact of different intervention strategies on the dynamics of oncogenic HPV-TB co-infections.

\subsection{Uncertainty and sensitivity analyses}

The oncogenic HPV-TB co-infection model (1) has thirty nine (39) parameters, and uncertainties are expected to arise in the estimates of their values used in the numerical simulations. Adopting the approach used by Blower and Dowlatabadi [3], we shall carry out a Latin Hypercube Sampling (LHS) on the parameters of the model. For the sensitivity analysis, a Partial Rank Correlation Coefficient (PRCC) was calculated between values of the parameters in the response function and the values of the response function derived from the sensitivity analysis. A total of 1,000 simulations of the co-infection model (1) per LHS were run. Using the total number of individuals dually infected with HPV and latent TB $\left(I_{\mathrm{HE}}^{\mathrm{P}}\right)$ as the response function, the parameters that strongly influence the dynamics of the co-infection model (1) are the demographic parameter, $\mu_{\mathrm{H}}$, the effective contact rate for HPV transmission, $\beta_{\mathrm{HP}}$, the parameter accounting for increased susceptibility to HPV by TB infected individuals, $\varrho_{1}^{\mathrm{P}}$, and the recovery rate from HPV for individuals in $I_{\mathrm{HE}}^{\mathrm{P}}$ compartment. Also, using the population of individuals dually infected with HPV and active TB $\left(I_{\mathrm{HA}}^{\mathrm{P}}\right)$ as the input, the five top ranked parameters are the effective contact rate for HPV transmissibility, $\beta_{\mathrm{HP}}$, the effective contact rate neccesary for TB transmission, $\beta_{\mathrm{T}}$, the modification parameter accounting for increased susceptibility to HPV infection by active TB infected individuals, $\varrho_{2}^{\mathrm{P}}$, the recovery rate from HPV for individuals in $I_{\mathrm{HA}}^{\mathrm{P}}$ compartment and the recovery rate from TB for individuals in $I_{\mathrm{HA}}^{\mathrm{P}}$ class. Taking the population of individuals dually infected with persistent HPV and latent TB $\left(P_{\mathrm{HE}}^{\mathrm{P}}\right)$ as the input, the three top ranked parameters are the effective contact rate for HPV transmissibility, $\beta_{\mathrm{HP}}$, the fraction of individuals who recover from HPV infection and do not progress to persistent HPV infection, $\theta_{2}^{\mathrm{P}}$, and the recovery rate from persistent HPV infection, $\rho_{3}^{\mathrm{P}}$, for individuals in $P_{\mathrm{HE}}^{\mathrm{P}}$ compartment. When the population of individuals dually infected with persistent HPV and active $\mathrm{TB}$ is used as the response function, the two top-ranked parameters are the effctive contact rate for TB transmission, $\beta_{\mathrm{T}}$ and the TB treatment rate, $r_{3}^{\mathrm{T}}$, for individuals dually infected with persistent HPV and active TB.

Considering the HPV associated basic reproduction number, $\mathcal{R}_{0 \mathrm{H}}$, as the response function, it is observed in Table 3 
medRxiv preprint doi: https://doi.org/10.1101/2020.09.15.20195297; this version posted September $27,2020$. The copyright holder for this preprint (which was not certified by peer review) is the author/funder, who has granted medRxiv a license to display the preprint in It is made available under a CC-BY-NC-ND 4.0 International license .

that the top four PRCC-ranked parameters are the demographic parameter, $\mu_{\mathrm{H}}$, effective contact rate for HPV transmission, $\beta_{\mathrm{HP}}$, the fraction of infected individuals who recover from HPV and do not develop persistent HPV, $\theta_{1}^{\mathrm{P}}$ and the recovery rate from HPV $\phi_{1}^{\mathrm{P}}$. Finally, using the TB associated reproduction number, $\mathcal{R}_{0 \mathrm{~T}}$, as the response function the two key parameters that drive the dynamics of the model are the effective contact rate for TB transmission, $\beta_{\mathrm{T}}$ and the TB treatment rate $r_{1}^{\mathrm{T}}$. 
medRxiv preprint doi: https://doi.org/10.1101/2020.09.15.20195297; this version posted September 27, 2020. The copyright holder for this preprint (which was not certified by peer review) is the author/funder, who has granted medRxiv a license to display the preprint in

It is made available under a CC-BY-NC-ND 4.0 International license.

Table 3: PRCC values for the co-infection model (1) parameters using the total number of individuals dually infected with: HPV and latent TB $\left(I_{\mathrm{HE}}^{\mathrm{P}}\right), \mathrm{HPV}$ and active TB $\left(I_{\mathrm{HA}}^{\mathrm{P}}\right)$, Persistent HPV and latent TB $\left(P_{\mathrm{HE}}^{\mathrm{P}}\right)$, Persistent HPV and active TB $\left(P_{\mathrm{HA}}^{\mathrm{P}}\right)$, respectively, as well as the associated reproduction numbers $\mathcal{R}_{0 \mathrm{H}}$ and $\mathcal{R}_{0 \mathrm{~T}}$, as response functions. Paramters which strongly influence the dynamics of the co-infection model with respect to each of the response functions are shown in bold fonts.

\begin{tabular}{|c|c|c|c|c|c|c|}
\hline Parameters & $I_{\mathrm{HE}}^{\mathrm{P}}$ & $I_{\mathrm{HA}}^{\mathrm{P}}$ & $P_{\mathrm{HE}}^{\mathrm{P}}$ & $P_{\mathrm{HA}}^{\mathrm{P}}$ & $\mathcal{R}_{0 \mathrm{H}}$ & $\mathcal{R}_{0 \mathrm{~T}}$ \\
\hline$\mu_{\mathrm{H}}$ & -0.4468 & -0.1478 & -0.3209 & 0.1208 & -0.5074 & -0.2068 \\
\hline$\beta_{\mathrm{HP}}$ & 0.9304 & 0.6982 & 0.6390 & 0.3300 & 0.9165 & - \\
\hline$\beta_{\mathrm{T}}$ & 0.3353 & 0.5813 & -0.0719 & 0.5620 & - & 0.8182 \\
\hline$\varrho_{1}^{\mathrm{P}}$ & 0.5387 & 0.0493 & 0.1298 & 0.0116 & - & - \\
\hline$\varrho_{2}^{\mathrm{P}}$ & 0.1403 & 0.5009 & 0.0683 & 0.1199 & - & - \\
\hline$\kappa_{\mathrm{T}}$ & 0.1835 & 0.2371 & 0.0167 & 0.0904 & - & - \\
\hline$\theta_{1}^{\mathrm{P}}$ & -0.3717 & -0.0286 & -0.1216 & -0.0579 & -0.7015 & - \\
\hline$\theta_{2}^{\mathrm{P}}$ & 0.1408 & 0.0140 & -0.7032 & -0.0782 & - & - \\
\hline$\theta_{3}^{\mathrm{P}}$ & 0.0097 & 0.0652 & 0.0233 & -0.2433 & - & - \\
\hline$\phi_{1}^{\mathrm{P}}$ & -0.1802 & -0.0323 & -0.0447 & -0.0534 & -0.8041 & - \\
\hline$\phi_{2}^{\mathrm{P}}$ & -0.6866 & -0.4079 & -0.2726 & -0.0732 & - & - \\
\hline$\phi_{3}^{\mathrm{P}}$ & 0.0394 & -0.2870 & -0.0175 & 0.2939 & - & - \\
\hline$r_{1}^{\mathrm{T}}$ & -0.0480 & -0.0471 & 0.000319 & 0.1303 & - & -0.5629 \\
\hline$r_{2}^{1}$ & 0.0805 & -0.8312 & 0.2174 & 0.1088 & - & - \\
\hline$r_{3}^{\mathrm{T}}$ & 0.0477 & 0.1487 & 0.3076 & -0.8994 & - & - \\
\hline$\rho_{1}^{\mathrm{P}}$ & -0.0891 & 0.0035 & -0.0376 & -0.0340 & - & - \\
\hline$\rho_{2}^{\mathrm{P}}$ & 0.1337 & 0.0170 & 0.0631 & 0.0208 & - & - \\
\hline$\rho_{3}^{\mathrm{P}}$ & -0.0734 & -0.0162 & -0.5220 & -0.3034 & - & - \\
\hline$\varphi_{\mathrm{P}}$ & 0.0443 & -0.0039 & 0.0061 & -0.0184 & - & - \\
\hline$\delta_{\mathrm{HP}}$ & 0.0120 & 0.0144 & 0.0208 & 0.0337 & -0.0478 & - \\
\hline$\delta_{\mathrm{HP} 1}$ & 0.0220 & -0.0026 & -0.0104 & 0.0085 & - & - \\
\hline$\delta_{\mathrm{HP} 2}$ & -0.0418 & -0.0033 & -0.0214 & 0.0213 & - & - \\
\hline$\delta_{\mathrm{T}}$ & 0.0275 & -0.0132 & 0.0270 & -0.0041 & - & -0.1606 \\
\hline$\delta_{\mathrm{T} 1}$ & 0.0016 & -0.1478 & 0.0101 & 0.0232 & - & - \\
\hline$\delta_{\mathrm{T} 2}$ & 0.0097 & 0.0232 & 0.0324 & -0.1941 & - & - \\
\hline$\sigma_{\mathrm{T}}$ & 0.0062 & -0.0006321 & 0.0229 & -0.00886 & - & - \\
\hline$\xi_{1}^{\mathrm{T}}$ & -0.0687 & 0.0941 & -0.0301 & 0.0407 & - & 0.3606 \\
\hline$\xi_{2}^{\mathrm{T}}$ & 0.0377 & 0.0611 & 0.3845 & 0.0232 & - & - \\
\hline$\xi_{3}^{\mathrm{T}}$ & 0.0077 & -0.0035 & -0.0143 & 0.0111 & - & - \\
\hline$\varepsilon_{1}^{\mathrm{T}}$ & -0.0116 & 0.0231 & -0.0132 & -0.0138 & - & - \\
\hline$\varepsilon_{2}^{\mathrm{T}}$ & 0.0052 & 0.0445 & 0.0188 & 0.047 & - & - \\
\hline$\varepsilon_{3}^{\mathrm{T}}$ & 0.0058 & 0.0202 & -0.0116 & 0.0927 & - & - \\
\hline$\gamma_{1}^{\mathrm{T}}$ & -0.0297 & 0.0089 & 0.049 & 0.0153 & - & 0.0793 \\
\hline$\gamma_{2}^{\mathrm{T}}$ & -0.0237 & 0.0842 & -0.0314 & -0.0003668 & - & - \\
\hline$\gamma_{3}^{\mathrm{T}}$ & -0.0127 & -0.0199 & -0.0326 & 0.0583 & - & - \\
\hline$\eta_{\mathrm{T}}$ & 0.0731 & 0.1261 & 0.0344 & 0.0318 & - & - \\
\hline$\eta_{\mathrm{P}}$ & 0.1506 & 0.1929 & 0.0470 & 0.1196 & - & - \\
\hline$\omega_{\mathrm{P}}$ & 0.0475 & 0.1403 & -0.0277 & 0.1404 & - & - \\
\hline
\end{tabular}


medRxiv preprint doi: https://doi.org/10.1101/2020.09.15.20195297; this version posted September 27, 2020. The copyright holder for this preprint (which was not certified by peer review) is the author/funder, who has granted medRxiv a license to display the preprint in

It is made available under a CC-BY-NC-ND 4.0 International license .

\section{$5.2 \quad$ Numerical simulations}

Numerical simulations of the optimal control problem [12, adjoint equations 18] and characterizations of the control 21] are implemented by the Runge Kutta method using the forward backward sweep (carried out in MATLAB). Following the choice of the weight function for HPV vaccination control used in Malik et al [17, the balancing factor $\chi_{1}=10^{3}$. We also assume $\chi_{2}=500$ and $\chi_{3}=400$. The demographic data related to the Shanxi province in rural China is used 8. We assume the initial conditions to be: $S(0)=1000, I_{\mathrm{HP}}(0)=300, P_{\mathrm{HP}}(0)=200, R_{\mathrm{HP}}(0)=200, E_{\mathrm{T}}(0)=600, A_{\mathrm{T}}(0)=$ $400, T_{\mathrm{T}}(0)=400, I_{\mathrm{HE}}^{\mathrm{P}}(0)=300, I_{\mathrm{HA}}^{\mathrm{P}}(0)=400, P_{\mathrm{HE}}^{\mathrm{P}}(0)=400, P_{\mathrm{HA}}^{\mathrm{P}}(0)=450$, . We implement the following three different control strategies for numerical simulations of the co-infection model (4). The unit of time is years, throughout the entire simulations.

i. Strategy A: Control against incident HPV infection $\left(u_{1} \neq 0\right)$ and control against infection with HPV by TB infected individuals $\left(u_{2} \neq 0\right)$;

ii. Strategy B: Control against incident HPV infection $\left(u_{1} \neq 0\right)$ and TB treatment control for dually infected individuals $\left(u_{3} \neq 0\right)$

iii. Strategy C: control against infection with HPV by TB infected individuals $\left(u_{2} \neq 0\right)$ and TB treatment control for dually infected individuals $\left(u_{3} \neq 0\right)$.

\subsubsection{Strategy A: Control against incident HPV infection $\left(u_{1} \neq 0\right)$ and control against infection with HPV by TB infected individuals $\left(u_{2} \neq 0\right)$}

Applying this control strategy, we note that the total number of individuals dually infected with HPV and TB in latent and active stages, respectively, (Figures 2 (a) and 2(b)), and the total number of individuals dually infected with persistent HPV and TB in latent and active stages, respectively (Figures 2(c) and 2(d)) are less when this control is applied than when the control is not applied. To be specific, this control strategy averts almost 420,352 new co-infection cases, which is the greatest number of averted cases in comparison with other control strategies implemented. The control profile for this strategy given in Figure 5 reveals that control $u_{1}$ is at its upper bound for the first 3.9 years before ultimately declining to zero. Similarly, the control $u_{2}$ is at the maximum value of $90 \%$ for the first 8 months before declining to zero at time, $t=4.5$ years.

\subsubsection{Strategy B: Control against incident HPV infection $\left(u_{1} \neq 0\right)$ and TB treatment control for dually infected individuals $\left(u_{3} \neq 0\right)$}

The simulations of the total number of dually infected individuals in the presence of TB treatment controls are depicted in Figures 3(a) -3(d). Applying this control, we observe that the total number of individuals dually infected with HPV and TB in latent and active stages of infection respectively, is less than the total population when no control is applied as expected (Figure 3(a) and 3(b)). Similarly, it is noticed from Figures [3.c) and 3(d), that high population level impact is observed in the total number of individuals dually infected with persistent HPV and TB in latent and active stages of infection, respectively, when this control strategy is applied. Specifically, when this control strategy is implemented, about 300,549 new co-infection cases were averted. The control profile for this strategy presented in Figure 7 shows that control $u_{1}$ is at its upper bound for the first 4.0 years before steadily declining to zero at final time. Also, the control $u_{3}$ is at the maximum value of $100 \%$ for the first 1.2 years and then steadily declines to zero at time, $t=4.5$ years. This simulation results conforms with the epidemiological report in the introduction section that gynaecologic TB is a risk factor for oncogenic HPV infection (and subsequent cancer infection) [37. Hence, if we focus on TB treatment controls, it can significantly reduce the burden of the co-infection of oncogenic HPV and TB in a population. The simulations are also in line with the point opined by 37, that prior TB infection was associated with persistent HPV and increased susceptibility to cervical cancer. As a result, treating TB infections in dually infected individuals will significantly curb the mixed infections of both diseases. 
medRxiv preprint doi: https://doi.org/10.1101/2020.09.15.20195297; this version posted September 27, 2020. The copyright holder for this preprint (which was not certified by peer review) is the author/funder, who has granted medRxiv a license to display the preprint in

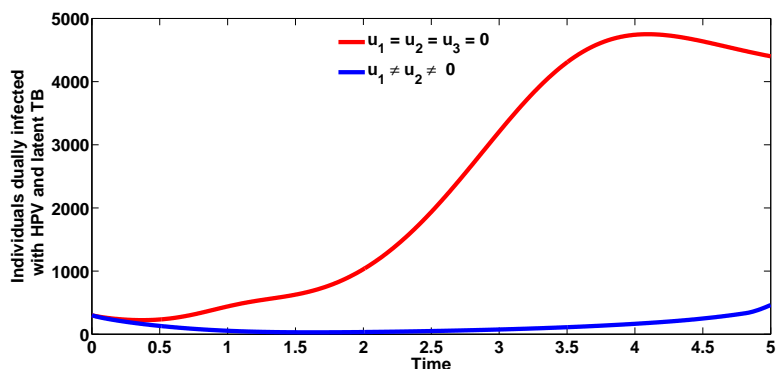

(a)

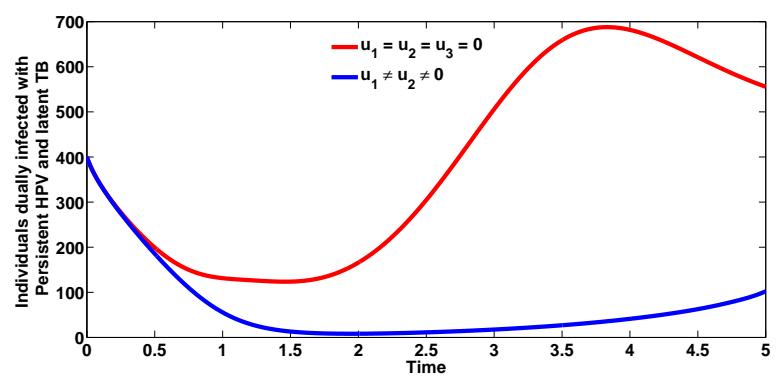

(c)

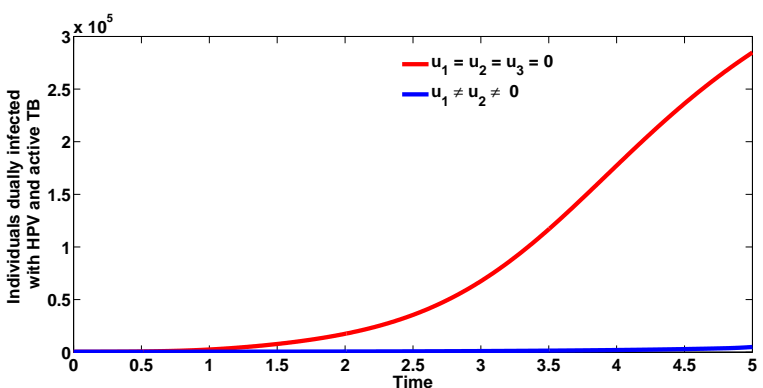

(b)

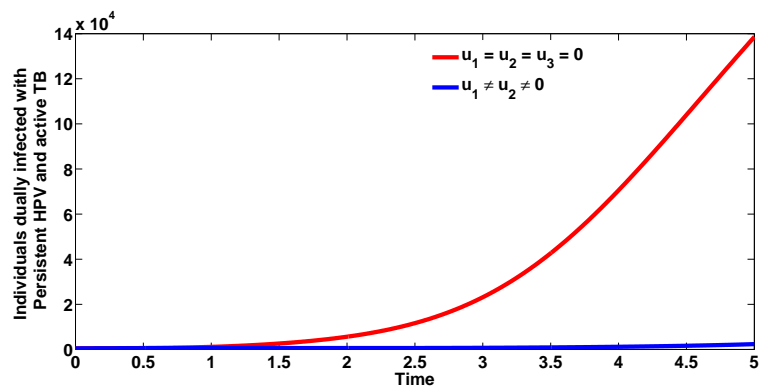

(d)

Figure 2: Plots of the total number of individuals dually infected with HPV and TB in latent and active stages, respectively (Figures $2 \mathrm{a}$ and $2 \mathrm{~b}$ ) as well as total number of individuals dually infected with persistent HPV and TB in latent and active stages (Figures 2 $c$ and 2 $\mathrm{d}$ ), respectively, the presence of optimal vaccination control against incident HPV infection $\left(u_{1} \neq 0\right)$ and control against infection with HPV by TB infected individuals $\left(u_{2} \neq 0\right)$. Here, $\beta_{\mathrm{HP}}=2.0, \beta_{\mathrm{T}}=8.557$. All other parameters as in Table 2

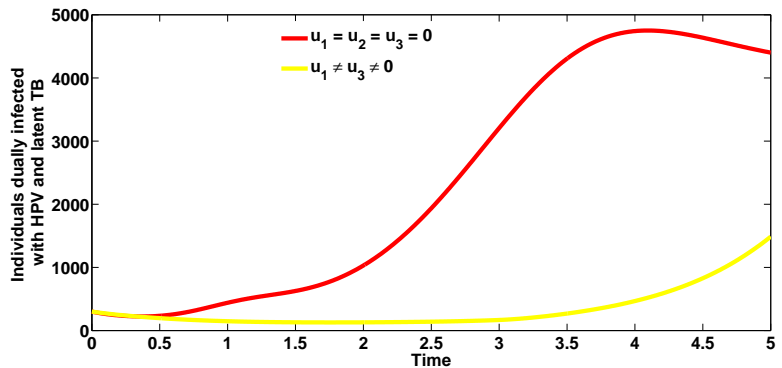

(a)

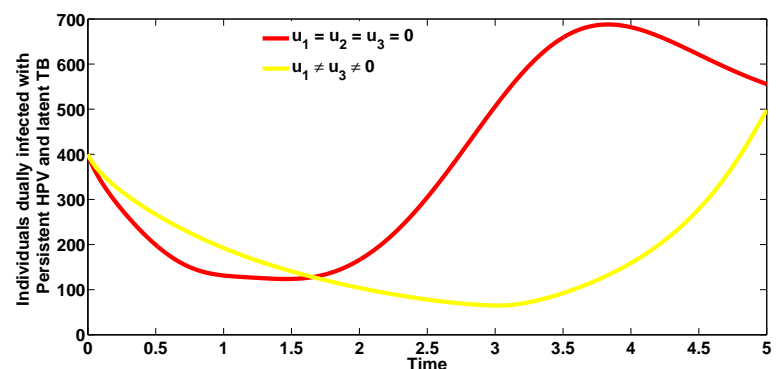

(c)

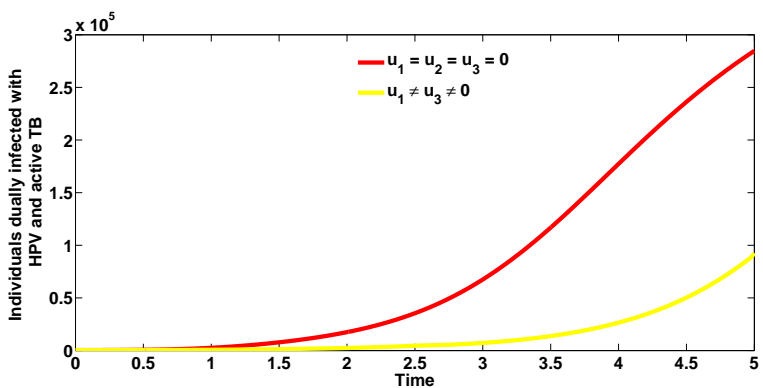

(b)

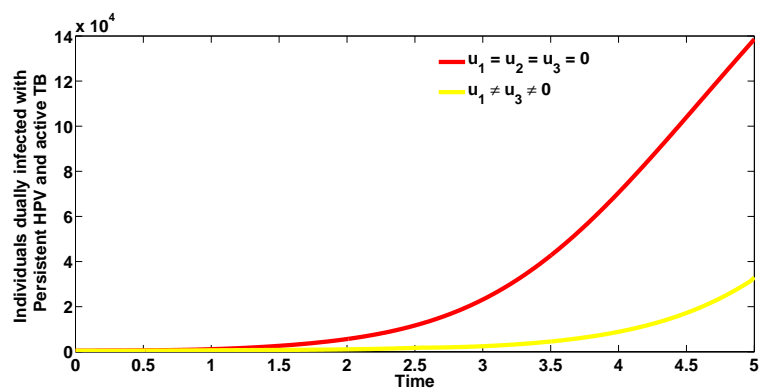

(d)

Figure 3: Plots of the total number of individuals dually infected with HPV and TB in latent and active stages, respectively (Figures 3(a) and 3(b) as well as total number of individuals dually infected with persistent HPV and TB in latent and active stages (Figures 3(c) and 3(d), respectively, the presence of optimal vaccination control against incident HPV infection $\left(u_{1} \neq 0\right)$ and TB treatment control dually infected individuals $\left(u_{3} \neq 0\right)$. Here, $\beta_{\mathrm{HP}}=2.0, \beta_{\mathrm{T}}=8.557$. All other parameters as in Table 2 
medRxiv preprint doi: https://doi.org/10.1101/2020.09.15.20195297; this version posted September $27,2020$. The copyright holder for this preprint (which was not certified by peer review) is the author/funder, who has granted medRxiv a license to display the preprint in It is made available under a CC-BY-NC-ND 4.0 International license

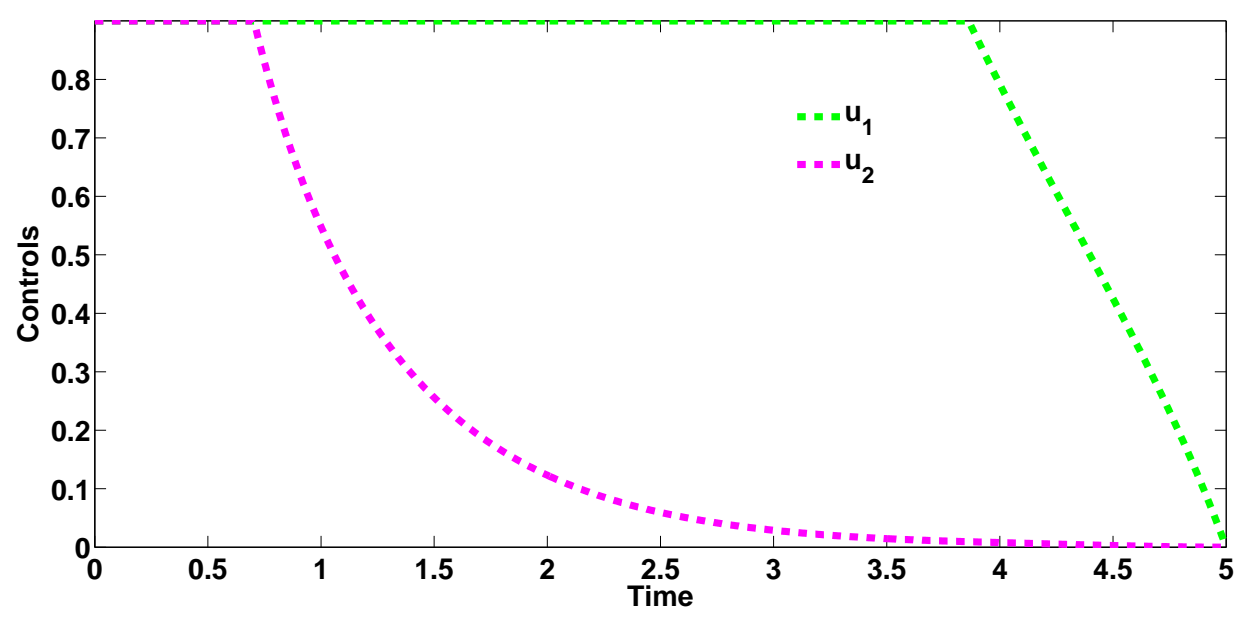

Figure 4

Figure 5: Combined effects of optimal controls $u_{1}$ and $u_{2}$ on the dynamics of the co-infection optimal control model $(12)$

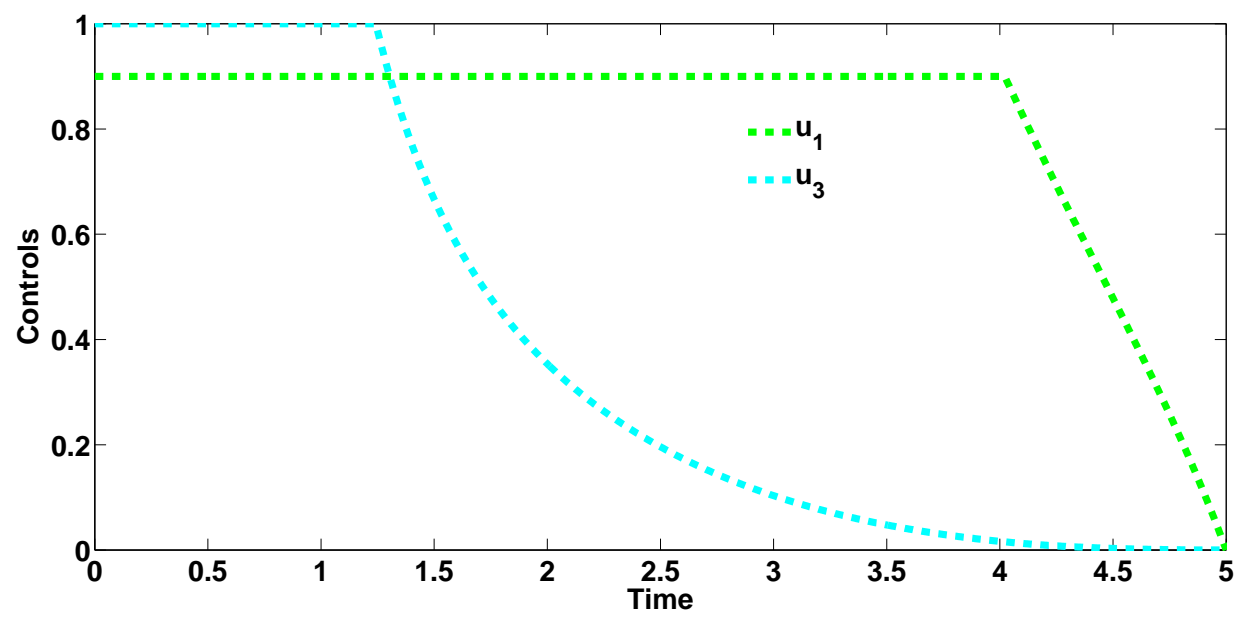

Figure 6

Figure 7: Combined effects of optimal controls $u_{1}$ and $u_{3}$ on the dynamics of the co-infection optimal control model (12) 
medRxiv preprint doi: https://doi.org/10.1101/2020.09.15.20195297; this version posted September 27, 2020. The copyright holder for this preprint (which was not certified by peer review) is the author/funder, who has granted medRxiv a license to display the preprint in It is made available under a CC-BY-NC-ND 4.0 International license.

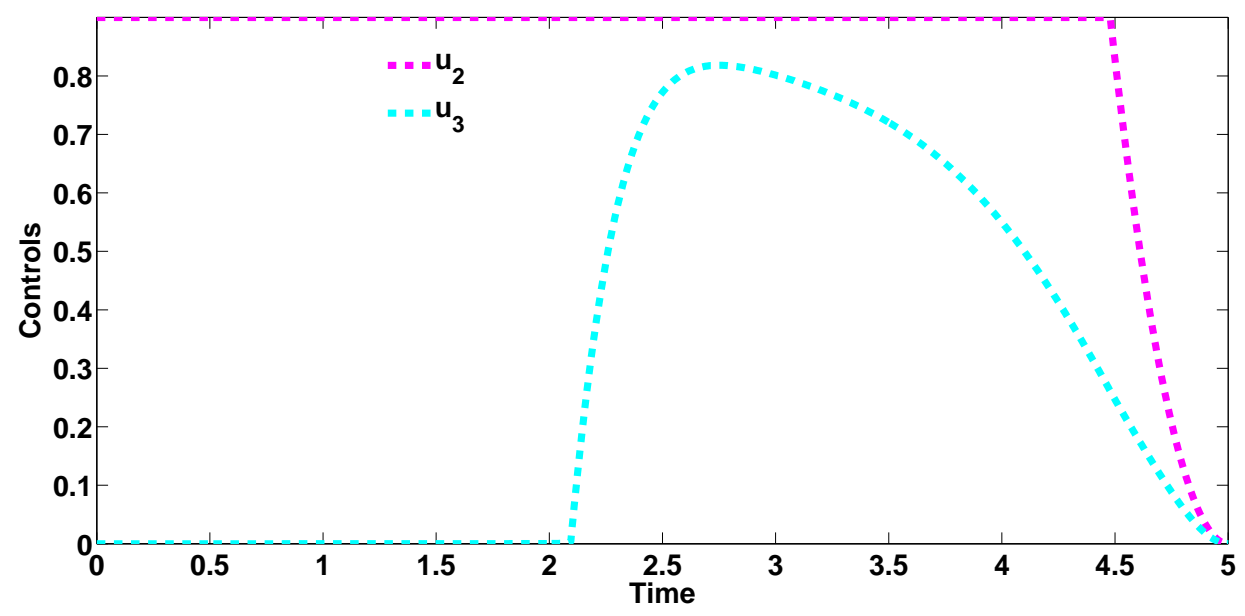

Figure 8

Figure 9: Combined effects of optimal controls $u_{2}$ and $u_{3}$ on the dynamics of the co-infection optimal control model 12

\subsubsection{Strategy C: control against infection with HPV by TB infected individuals $\left(u_{2} \neq 0\right)$ and TB treatment control for dually infected individuals $\left(u_{3} \neq 0\right)$}

Using this control strategy, we observe in Figures 10 (a) and 10 (b), that the number of individuals dually infected with HPV and TB in latent and active stages of infection, respectively, is less than the number when no control strategy is applied. Likewise, this strategy has positive population level impact on the populations of infected individuals dually infected with persistent HPV and TB in latent and active stages of infection, respectively, Figures 10(c) and 10 (d). When this intervention strategy is administered, approximately 362,573 new co-infection cases are averted. The control profile for this strategy, depicted by Figure 9 reveals that control $u_{2}$ is at its upper bound for the first 4.5 years before gradually falling down to zero at final time. Moreso, the control $u_{3}$ is at its minimum value for the first 2.1 years and then steadily rises to its peak value of $100 \%$ at time, $t=2.7$ years, before gradually declining to zero at final time. It is imperative to state categorically, that the results of the simulations are based on the parameter values given in table 2 and the initial conditions and weight constants given in Section 5.2

\subsection{Cost-effectiveness analysis}

The cost-effectiveness analysis is used to evaluate the health interventions related benefits so as to justify the costs of the strategies [4]. This is obtained by comparing the differences among the health outcomes and costs of those interventions; achieved by computing the incremental cost-effectiveness ratio (ICER), which is defined as the cost per health outcome. It is given by:

$$
\text { ICER }=\frac{\text { Difference in costs between strategies }}{\text { Difference in health effects between strategies }} .
$$

We calculated the total number of co-infection cases averted and the total cost of the strategies applied in Table 4 The total number of co-infection cases prevented is obtained by calculating the total number of individuals when controls are administered and the total number when control is not applied. Similarly, we apply the cost functions $\frac{1}{2} \chi_{1} u_{1}^{2}, \frac{1}{2} \chi_{2} u_{2}^{2}, \frac{1}{2} \chi_{3} u_{3}^{2}$, over time, to compute the total cost for the various strategies that we implemented. We now compare the cost-effectiveness of strategy B (Optimal HPV vaccination control for sexually active susceptible individuals against incident HPV infection and TB treatment control for dually infected individuals) and strategy C (Control against HPV infection by TB infected 


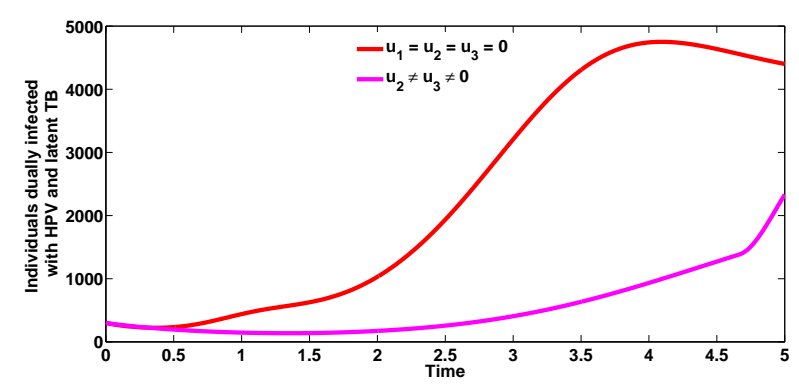

(a)

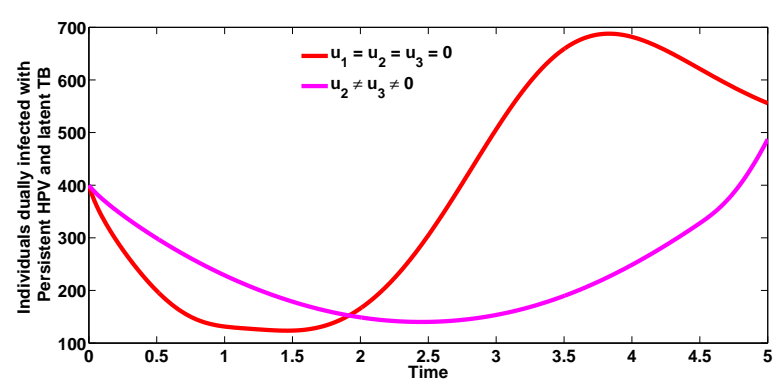

(c)

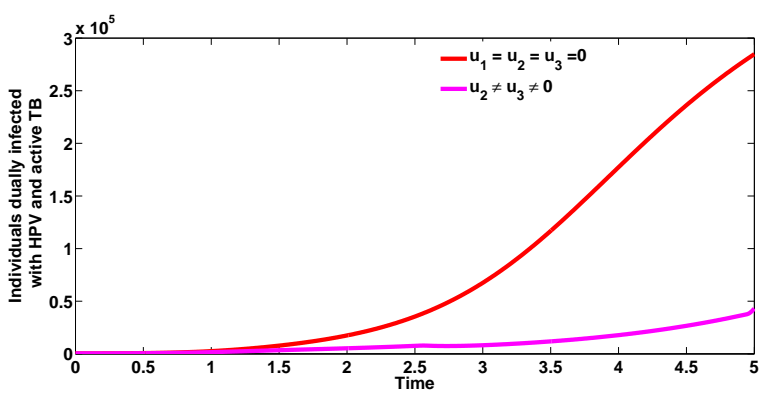

(b)

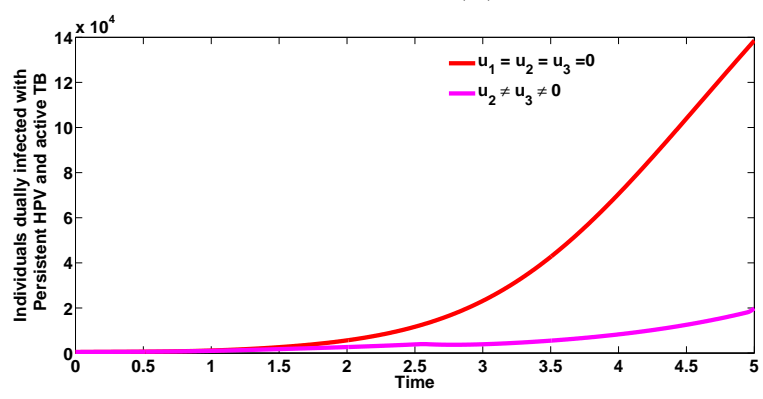

(d)

Figure 10: Plots of the total number of individuals dually infected with HPV and TB in latent and active stages, respectively (Figures $10 \mathrm{a}$ and $10 \mathrm{~b}$ ) as well as total number of individuals dually infected with persistent HPV and TB in latent and active stages (Figures 10c and 10 d), respectively, in the presence of control against HPV infection by TB-infected individuals $\left(u_{2} \neq 0\right)$ and TB treatment control dually infected individuals $\left(u_{3} \neq 0\right)$. Here, $\beta_{\mathrm{HP}}=2.0, \beta_{\mathrm{T}}=8.557$. All other parameters as in Table 2

individuals and TB treatment controls for dually infected individuals).

$$
\begin{aligned}
\operatorname{ICER}(B) & =\frac{1210}{300,549.2}=0.004026 \\
\operatorname{ICER}(C) & =\frac{405-1210}{362,573.4-300,549.2}=-0.01298
\end{aligned}
$$

From ICER (B) and ICER(C), we observe a cost saving of 0.01298 observed for strategy C over strategy B. This implies that strategy B strongly dominated strategy C, showing that strategy B is more costly and less effective compared to strategy C. Therefore, strategy B is removed from subsequent ICER computations, shown in Table 5 . We shall now compare strategies $\mathrm{C}$ and A. Comparing strategy C (Control against HPV infection by TB infected individuals and TB treatment controls

Table 4: Increasing order of the total infection averted due to the control strategies

\begin{tabular}{lllll}
\hline Strategy & Total infection averted & Total cost & ACER & ICER \\
\hline B: $u_{1}(t), u_{2}(t)$ & $300,549.2$ & 1,210 & 0.004026 & 0.004026 \\
C: $u_{2}(t), u_{3}(t)$ & $362,573.4$ & 405 & 0.001117 & -0.01298 \\
A: $u_{1}(t), u_{2}(t)$ & $420,352.2$ & 1,215 & 0.002890 & 0.01402 \\
\hline
\end{tabular}

Table 5: Increasing order of the total infection averted due to the control strategies

\begin{tabular}{lllll}
\hline Strategy & Total infection averted & Total cost & ACER & ICER \\
\hline C: $u_{2}(t), u_{3}(t)$ & $362,573.4$ & 405 & 0.001117 & 0.001117 \\
A: $u_{1}(t), u_{2}(t)$ & $420,352.2$ & 1,215 & 0.002890 & 0.01402 \\
\hline
\end{tabular}

for dually infected individuals) and strategy A (Optimal HPV vaccination control for sexually active susceptible individuals 
medRxiv preprint doi: https://doi.org/10.1101/2020.09.15.20195297; this version posted September 27, 2020. The copyright holder for this preprint (which was not certified by peer review) is the author/funder, who has granted medRxiv a license to display the preprint in

It is made available under a CC-BY-NC-ND 4.0 International license .

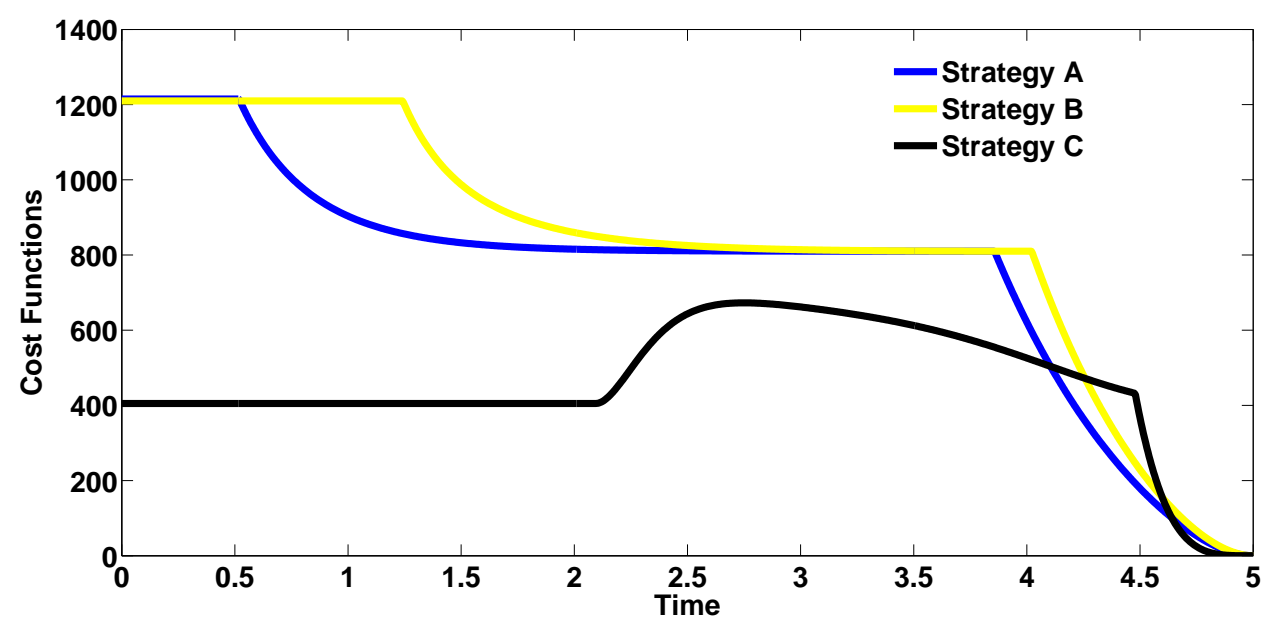

Figure 11

Figure 12: Cost functions of the different control strategies

against incident HPV infection and Control against HPV infection by TB infected individuals), we observe that ICER (A) is greater than ICER (C), showing that strategy A strongly dominated strategy C and is more expensive and less effective compared to strategy C. Consequently, strategy C (the strategy that combines and implements control against HPV infection by TB infected individuals as well as TB treatment controls for dually infected individuals) has the least ICER and is the most cost-effective of all the control strategies for the control and management of oncogenic HPV and TB co-infection. This clearly agrees with the results obtained from ACER method in Table 4 that strategy $\mathrm{C}$ is the most cost-effective strategy.

$$
\begin{aligned}
\operatorname{ICER}(\mathrm{C}) & =\frac{405}{362,573.4}=0.001117 \\
\operatorname{ICER}(\mathrm{A}) & =\frac{1215-405}{420,352.2-362,573.4}=0.01402
\end{aligned}
$$

\section{Conclusion}

In this work, we have developed and presented a co-infection model for Oncogenic HPV and TB with cost-effectiveness optimal control analysis. The full co-infection model was shown to undergo the phenomenon of backward bifurcation when the associated reproduction number is less than unity. It was further shown that TB and HPV re-infection parameters $\left(\varphi_{\mathrm{P}} \neq 0\right.$ and $\left.\sigma_{\mathrm{T}} \neq 0\right)$ as well as the exogenous re-infection term $\left(\varepsilon_{1} \neq 0\right)$ induced the phenomenon of backward bifurcation in the oncogenic HPV-TB co-infection model. The global asymptotic stability of the disease-free equilibrium of the coinfection model was also proven not to exist, when the associated reproduction number was below unity. The necessary conditions for the existence of optimal control and the optimality system for the co-infection model was established using the Pontryagin's Maximum Principle. Uncertainty and global sensitivity analyses were also carried out to determine the top ranked parameters that influence the dynamics of the co-infection model, when the associated reproduction numbers as well as the infected populations were used as response functions. When the population of individuals dually infected with $\mathrm{HPV}$ and latent TB $\left(I_{\mathrm{HE}}^{\mathrm{P}}\right)$ was used as the response function, the parameters that strongly influence the dynamics of the co-infection model (1) are the demographic parameter, $\mu_{\mathrm{H}}$, the effective contact rate for HPV transmission, $\beta_{\mathrm{HP}}$, the parameter accounting for increased susceptibility to HPV by TB infected individuals, $\varrho_{1}^{\mathrm{P}}$, and the recovery rate from HPV for individuals in $I_{\mathrm{HE}}^{\mathrm{P}}$ compartment. In addition, using the population of individuals dually infected with HPV and active TB $\left(I_{\mathrm{HA}}^{\mathrm{P}}\right)$ as the input, the five top ranked parameters are the effective contact rate for HPV transmissibility, $\beta_{\mathrm{HP}}$, the effective contact rate neccesary for TB transmission, $\beta_{\mathrm{T}}$, the modification parameter accounting for increased susceptibility to HPV infection by active TB infected individuals, $\varrho_{2}^{\mathrm{P}}$, the recovery rate from HPV for individuals in $I_{\mathrm{HA}}^{\mathrm{P}}$ compartment and the 
medRxiv preprint doi: https://doi.org/10.1101/2020.09.15.20195297; this version posted September 27, 2020. The copyright holder for this preprint (which was not certified by peer review) is the author/funder, who has granted medRxiv a license to display the preprint in It is made available under a CC-BY-NC-ND 4.0 International license .

recovery rate from TB for individuals in $I_{\mathrm{HA}}^{\mathrm{P}}$ class. Taking the population of individuals dually infected with persistent HPV and active TB as the response function, the two top-ranked parameters are the effctive contact rate for TB transmission, $\beta_{\mathrm{T}}$ and the TB treatment rate, $r_{3}^{\mathrm{T}}$, for individuals dually infected with persistent HPV and active TB.

Numerical simulations of the optimal control model showed that:

i. A combination of HPV prevention control and TB treatment control has a positive population level impact in reducing the burden of oncogenic HPV and TB co-infection cases in a population.

ii. A strategy that implements control against HPV infection by TB infected individuals and TB treatment controls for dually infected individuals can significantly reduce the burden of oncogenic HPV and TB co-infections.

iii. The strategy that combines and implements control against HPV infection by TB infected individuals as well as TB treatment control for dually infected individuals has the least ICER and is the most cost-effective of all the control strategies for the control and management of the burden of oncogenic HPV and TB co-infection. 
medRxiv preprint doi: https://doi.org/10.1101/2020.09.15.20195297; this version posted September 27, 2020. The copyright holder for this preprint (which was not certified by peer review) is the author/funder, who has granted medRxiv a license to display the preprint in It is made available under a CC-BY-NC-ND 4.0 International license .

\section{References}

[1] FB Agusto, AI Adekunle, Optimal control of a two-strain tuberculosis-HIV/AIDS co-infection model, BioSystems, (2014) (119) 20-24.

[2] HG Ahmed, SH Bensumaidea, IM Ashankyty, Frequency of human papillomavirus (HPV) subtypes 31, 33, 35, 39 and 45 among Yemeni women with cervical cancer, Infect. Ag. Cancer 10 (29) (2015) 1-6.

[3] SM Blower, H Dowlatabadi, Sensitivity and uncertainty analysis of complex models of disease transmission: an HIV model, as an example, International Statistical Review 2 (1994) 229-243.

[4] SB Cantor, TG Ganiats, Incremental cost-effectiveness analysis: the optimal strategy depends on the strategy set. Clin. Epidemiol. 52 (1999) (6), 517-522.

[5] C Castillo-Chavez, Z Feng, W Huang, On the computation of $R_{0}$ and its role on global stability, in Mathematical Approaches for Emerging and Reemerging Infectious Diseases: An Introduction (Minneapolis, MN, 1999), 229-250, IMA Vol. Math. Appl., 125 Springer New York.

[6] C Castillo-Chavez, B Song, Dynamical models of tuberculosis and their applications, Math. Biosci. Eng. 2 (2004) 361-404.

[7] T Cohen, C Colijin, B Finklea, M Murray, Exogenous re-infection and the dynamics of tuberculosis epidemics: local effects in a network model of transmission, J. R. Soc. Interface, 4 (2007) 523-531.

[8] China Demographics Profile, 2018, Available at http://www.indexmundi.com/china/demographics_profile. Accessed: 2 May, 2018.

[9] Fatmawati, H Tasman, Optimal control of HIV resistance and tuberculosis co-infection using treatment intervention, Asian Pac. J. Trop. Dis. 7 (2017) 366-373.

[10] FM Gilbert, I Ihejirika, SC Inyama, A Omame, HI Mbachu, JI Uwakwe, Deterministic Mathematical Model of Tuberculosis Disease with Treatment and Recovered Groups, International Journal of Mathematics and Statistics Invention (2019) 7 (1), 47-60

[11] AO Isere, D Okuonghae, Optimal control model for the outbreak of cholera in Nigeria, African Journal of Mathematics and Computer Science Research, 7;24-30; 2014

[12] A Jemal, F Bray, MM Center, J Ferlay, E Ward, D Forman, Global Cancer Statistics, CA Cancer J. Clin. 61 (2011) 69-90.

[13] J. L. Kelley, General topology, Springer-Verlag, (1991) ISBN 978-0-387-90125-1

[14] S Lenhart, JT Workman, 2007. Optimal Control Applied to Biological Models. Chapman \& Hall, Boca Raton.

[15] A MacNeil, P Glaziou, C Sismanidis, S Maloney, K Floyd, Global Epidemiology of Tuberculosis and Progress Toward Achieving Global Targets - 2017. MMWR Morb Mortal Wkly Rep (2019) 68 263?266 DOI: http://dx.doi.org/10.15585/mmwr.mm6811a3

[16] MT Malik, J Reimer, AB Gumel, EH Elbasha, SM Mahmud, The impact of an imperfect vaccine and pap cytology screening on the transmission of Human Papillomavirus and occurrence of associated cervical dysplasia and cancer, Math. Biosci. Eng. 10 (4) (2013).

[17] T Malik, M Imran, R Jayaraman, Optimal control with multiple human papillomavirus vaccines, Journal of Theoretical Biology, (393) 179-193, 2016.

[18] J Mohammed-Awel, E Numfor, Optimal Insecticide Treated Bednets Coverage and Malaria Treatment in a Malaria-HIV Co-infection Model. Journal of Biological Dynamics, 11 (2017) - Issue sup1 : 160-191.

[19] Z Mukandavire, AB Gumel, W Garira, JM Tchuenche, Mathematical Analysis of a Model for HIV-Malaria Co-infection, Math. Biosci. Eng. 6 (2) (2009) 333-362.

[20] KO Okosun, OD Makinde, A co-infection model of malaria and cholera diseases with optimal control, Math. Biosci. 258 (2014) 19-32.

[21] D Okuonghae, A Korobeinikov, Dynamics of tuberculosis: the effect of direct observation therapy strategy (DOTS) in Nigeria, Math. Model. Nat. Phenomena, (2) (2007) 1 99-111.

[22] D Okuonghae, V Aihie, Case detection and direct observation therapy strategy (DOTS) in Nigeria: its effect on TB dynamics, J. Biol. Syst. 16 (1) (2008) 1-31.

[23] A Omame, RA Umana, D Okuonghae, SC Inyama, Mathematical analysis of a two-sex Human Papillomavirus (HPV) model, International Journal of Biomathematics, 11 (2018) (7) 
medRxiv preprint doi: https://doi.org/10.1101/2020.09.15.20195297; this version posted September 27, 2020. The copyright holder for this preprint (which was not certified by peer review) is the author/funder, who has granted medRxiv a license to display the preprint in

It is made available under a CC-BY-NC-ND 4.0 International license .

[24] A Omame, D Okuonghae, RA Umana, SC Inyama, Analysis of a co-infection model for HPV-TB, Applied Mathematical Modelling, 77 (2020) 881-901.

[25] A Omame, D Okuonghae, SC Inyama, A mathematical study of a model for HPV with two high risk strains, in Mathematics Applied to Engineering, Modelling, and Social Issues Studies in Systems, Decision and Control (2020) 200, F. Smith, H. Dutta and J. N. Mordeson (eds.)

[26] A Omame, N Sene, I Nometa, CI Nwakanma, EU Nwafor, NO Iheonu, D Okuonghae, Analysis of COVID-19 and comorbidity co-infection Model with Optimal Control, medRxiv preprint (2020) doi: https://doi.org/10.1101/2020.08.04.20168013.

[27] A Omame, CU Nnanna, SC Inyama, Optimal Control and Cost-effectiveness Analysis of an HPV-Chlamydia Trachomatis co-infection model, medRxiv preprint (2020) doi:https://doi.org/10.1101/2020.09.07.20190025.

[28] LS Pontryagin, VG Boltyanskii, RV Gamkrelidze, EF Mishchenko, The Mathematical Theory of Optimal Processes, Wiley, New York, 1962.

[29] F Saldana, A Korobeinikov, I Barradas, Optimal Control against the Human Papillomavirus: Protection versus Eradication of the Infection, Abstract and Applied Analysis, https://doi.org/10.1155/2019/4567825

[30] Shanxi Demographics Data, 2015, Available at http://www.khoema.com/atlas/china/shanxi Accessed: 2 May, 2018.

[31] Tanvi, R Aggarwal, Dynamics of HIV-TB co-infection with detection as optimal intervention strategy, International Journal of Non-Linear Mechanics (2020), doi:https://doi.org/10.1016/j.ijnonlinmec.2019.103388.

[32] GT Tilahun, OD Makinde, D Malonza, Co-dynamics of Pneumonia and Typhoid fever diseases with cost effective optimal control analysis, Appl. Math. Comput. 316 (2018) 438-459.

[33] RA Umana, A Omame, SC Inyama, Deterministic and Stochastic Models of the Dynamics of Drug Resistant Tuberculosis; FUTO Journals Series, (2016) 2 (2) 173-194.

[34] JI Uwakwe, SC Inyama, A Omame, Mathematical Model and Optimal Control of New-Castle Disease (ND). Applied and Computational Mathematics. (2020) 9 (3) 70-84. doi: 10.11648/j.acm.20200903.14

[35] P van den Driessche, J Watmough, Reproduction numbers and sub-threshold endemic equilibria for compartmental models of disease transmission, Math. Biosci. 180 (2002) 29-48.

[36] World Health Organization. Global tuberculosis report 2018. Geneva, Switzerland: World Health Organization; 2018. https://www.who.int/tb/publications/global report/en

[37] NM Zetola, S Grover, C Modongo, SP Chiyapo, Collision of three pandemics: The coexistence of cervical cancer, HIV infection, and the prior tuberculosis in the sub-Saharan country of Botswana, J. Glob. Oncol. DOI:10.1200/JGO.2015.001701.

[38] S Zhu, L Xia, S Yu, S Chen, J Zhang, The burden and challenges of tuberculosis in China: findings from the Global Burden of Disease Study 2015, Nature, 7 (2017) 14601, DOI:10.1038/s41598-017-15024-1.

[39] FH Zhao, AP Varanasi, CA Cunningham, BI Graubard, S-Y Hu, F Chen, CJ Barrett, Y-L Qiao, MR Forman, Tuberculosis and oncogenic HPV: Potential co-infections in women at high-risk of cervical cancer in rural China. Asian P. J. Canc. Prev. 12 (2011) 1409-1415. 